\title{
LE PÉCULE ROMAIN ET L'ORIGINE DE LA RESPONSABILITE LIMITÉE EN DROIT ROMAIN
}

DOI: 10.24193 /SUBBiur.65(2020).4.20

Data publicării online:

16.03.2021

\section{Malina NOVKIRISHKA-STOYANOVA*}

Résumé: L'étude comprend une partie d'une étude plus approfondie sur le pécule romain des esclaves et des personae alieni iuris présenté dans son évolution. L'accent est mis principalement sur l'émergence de la responsabilité limitée du pater familias /dominus en cas de contrats conclus avec des biens péculiaires. On étudie la place de l'actio de peculio parmi les autres actiones adjectitiae qualitatis, la notion de la merx pexuliaris et la concessio peculii.

Mots clés : peculium, pater familias, dominus, alieni iuris, servi, actio de peculio, actio tributoria, merx pexuliaris.

* Docteur ès sciences juridiques, Université de Sofia « St. Clément d’Ohrid », Bulgarie malinans@uni-sofia.bg.

$$
672
$$




\title{
THE ROMAN PECULIUM AND THE ORIGIN OF LIMITED LIABILITY IN ROMAN LAW
}

\begin{abstract}
The study is a part of one deeper study on the Roman Law about the slaves and personae alieni iuris presented in its evolution. The accent is mainly on the emergence of the limited liability of the pater familias/ dominus in the case of contracts with pecuniary property. It is a study for the place of the actio de peculio among the other actiones adjectitiae qualitatis, the notion of the merx pexuliaris and the concessio particulii.
\end{abstract}

Keywords: peculium, pater familias, dominus, alieni iuris, servi, actio de peculio, actio tributoria, merx pexuliaris.

Table des matières

I. État de question 673

II. La notion de pécule comme pratique et réglementation juridique 678

A. La terminologie et l'origine. 678

B. Les sources. .682

III. Actio de peculio comme une des actiones adjectitiae qualitatis.............. 686

IV. Détermination et séparation patrimoniale du pécule ......................... 698

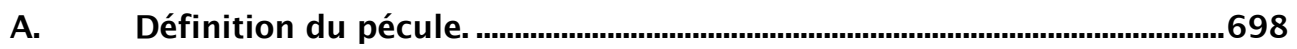

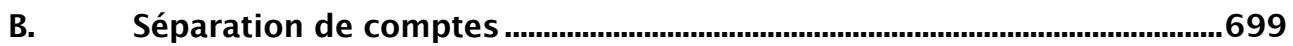

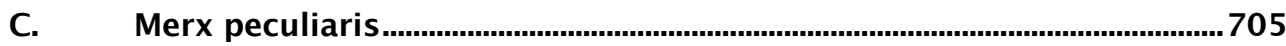

V. Concessio peculii et les modes de création du pecule. ....................... 719

VI. Conclusion ........................................................................... 724

\section{I. État de question}

Le problème du pécule en droit romain a depuis longtemps attiré l'attention des romanistes tant sur le plan historique que dogmatique. Malgré le nombre relativement volumineux d'ouvrages qui lui sont spécialement consacrés, il reste dans une certaine mesure énigmatique, en particulier si on 
le compare aux autres formes de participation d'esclaves et de alieni iuris aux rapports civils ${ }^{1}$.

Lorsqu'on se penche sur cet institut ${ }^{2}$ traditionnel pour la famille romaine, il va falloir prendre en considération son dynamisme et le situer correctement dans son milieu économique, social, politique et juridique, vu le moment auquel se réfère le régime juridique correspondant.

Le présent exposé est consacré au pécule et respectivement à la place de la responsabilité limitée du chef de famille pour des contrats conclus par

${ }^{1}$ Pour la littérature récente voir la bibliographie en PESARESI, R. Studi sull'actio de peculio Bari, 2012 ; ID. Ricerche sul peculium imprenditoriale. Bari, 2008. En général voir MANDRY, G. Das gemeine Familienguterrecht mit Ausschluss des ehelichem Guterrechtes. v.II. Das Profectizische Peculium. Tubingen 1876 ; MICOLIER, G. Pécule et capacité patrimoniale : Etude sur le pécule, dit profectice. Depuis l'édit de peculio jusqu'a la fin de l'époque classique. Lyon 1932 ; HERVIEUX, L. Des Pécules du fils de famille dans la législation romaine. Paris 1890; ZEBER, I. A study of the peculium of a slave in preclassical and classical Roman Law. Wroclaw 1981; BUTI, I.,Studi sulla capacità patrimoniale dei `servi` . Napoli 1976; RUGGIERO, E. Sulla 'libera administratio peculii '.- In: Rendiconti del R.Istituto Lombardo di Scienze e Lettere`. v. LXI. Fasc.XVI-XX. (1928). Milano 1929, p. 833-849. Pour l'actio de peculio voir LEMARIÉ, L. De l' Action tributoria 'ou de la Liquidation du Pécule commercial de l'Esclave Romain. Paris 1910; ALBERTARI, E. Responsabilità fino al limite dell'arricchimento nell' 'Actio Tributoria e nell' 'Actio De Peculio`. Pavia 1913; DE LUSIGNANI, L. La consumazione processuale dell' 'actio de peculio ` : Note esegetiche. Parma, 1889 ; SOLAZZI, S. `Jus deductionis`e `condemnatio cum deductione` nell' ‘actio de peculio`. Napoli, 1905. ; SECKEL, E. Die Haftung de peculio und de in rem verso aus der Litiskontestation und dem Urteil nach klassischem römischen Recht. Weimar 1907. Pour des pécules spéciales voir FITTING, H. Das castrnse peculium in seiner geschichtlichen Entwicklung. Halle 1871; LA ROSA, F. I peculii speciali in diritto romano. Milano 1953; MUEHLBERG, O. De peculio castrensi non retrotrahendo quum penes patrem iure peculii remanet : Dissertatio Inauguralis. Berlin 1886, ainsi que les etudes de APPLETON, CH. Les pouvoirs du fils de famille sur son pécule castrans et la date des Digesta de Julien. in: NRHDFE (1911). p. 593-623; ALBERTARIO, E. Appunti sul peculio castrense. Roma ; 1931; ARCHI, G. G. In tema di peculio quasi castrense. Citta di Castello, 1938.

${ }^{2}$ On utilise les notions contemporaines des normes de droit, institut juridique, sujet de droit etc. malgrè qu'en droit romain il existe de telles notions mais il n'y a pas de la terminologie correspondante. 
alieni iuris et esclaves avec le pécule3. On ne pourra bien évidemment trouver une continuité directe et une reproduction des principales caractéristique du pécule romain dans le système juridique actuel, dans la mesure où il est lié à une forme traditionnelle pour les sociétés antiques de dépendance des membres de la famille et des esclaves du chef de famille comme chef d'une grande famille patriarcale. C'est dans les systèmes juridiques antiques qu'il existe le "pouvoir du père » à Rome (patria potestas à l'égard des fils de famille et autres alieni iuris et dominium ou dominica potestas à l'égard des esclaves), le droit intervenant de façon restreinte et délicate dans la réglementation des rapports concernant les biens dans la grande famille patriarcale, qui dans la plupart des cas sont réglés selon la coutume juridique. C'est uniquement en droit romain que le modèle d'usage du potentiel effectif et les possibilités intellectuelles de ces personnes acquièrent une forme institutionnelle, se développe comme un institut juridique indépendant et prend une place considérable dans le système juridique romain 4 .

L'étude du pécule pourrait se faire sous divers aspects:

3 Il y a deux textes en Digeste où on utilise les locutions communes (personae in potestate) ainsi que pour les fils de famille (aussi des filles et des petits- enfants) que pour des esclaves qui toutes les deux catégories des personnes assez différentes reçoivent des pécules de son dominus ou pater familias : D. 15.1.1 (Ulpianus libro 29 ad edictum) 2. Verba autem edicti talia sunt: "Quod cum eo, qui in alterius potestate esset, negotium gestum erit"... 5. Potestatis verbum communiter accipiendum est tam in filio quam in servo. Mais dans la plupart des textes on parle séparément pour des fils et des esclaves ou on entend par analogie les normes pour les uns et les autres.

4 Pour des relations pater familias- filius familais et dominus- servus par rapport au pécule voir en manuels : ANDREEV, M. Droit romain privé (en bulgare : Андреев М.. Римско частно право). Sofia, 1975, p. с. 157. 177-178; BASANOV, I. Droit romain (en bulgare : Базанов И.. Римско право.). Vol. I. Sofia, 1940. p. 56 s.; GIRARD, P. F. Manuel élémentaire de Droit Romain. Paris,1924. p. 103,139-141; PETIT, E. Traité élémentaire de Droit Romain. Paris, 1906, p.68; 265 ; ELLUL, J. Histoire des Institutions.T.I. Paris,.1955, p.414 s.; OURLIAC P., MALAFOSSE J. DE. Histoire du droit privé. Droit famillial. Paris, 1968. p. 47.

\section{5}


1. en rapport avec l'esclavage et la participation des esclaves dans l'économie romaine, les rapports esclave-maitre etc. ;

2. en rapport avec l'évolution de la famille romaine comme communauté sociale et comme propriétaire de biens et la participation des alieni iuris aux rapports commerciaux;

3. dans le cadre de l'évolution générale et le passage de rapports agraires à des rapports économiques de marché;

4. en considération avec le dynamisme dans les échanges de marchandises au cours de cette évolution et la protection de tous les participants à ces échanges civils;

5. comme étape de l'évolution de l'intervention prétorienne dans le règlement de rapports liés au développement actuel de la société et de l'économie et non réglementés en droit civil;

6. comme forme utilisée pour des relations analogues en droit contemporain lorsqu'il s'agit de représentation, de représentation commerciale, de responsabilité pour les actions de tierces personnes, de responsabilité patrimoniale limitée etc.

Dans chacun de ces aspects on pourra observer la tendance spécifique en droit romain de mettre en place un régime juridique adéquat à des rapports qui, déjà établis dans la pratique, ont une influence positive pour le développement de la société, de l'économie et de l'État. C'est justement dans le pécule que l'on peut voir comment le droit brise le modèle traditionnel de l'esclavage et le cadre strict de la grande familia patriarcale et permet de se servir du potentiel personnel, intellectuel et des aptitudes d'affaires des esclaves et des alieni iuris tant dans l'intérêt de leur dominus/ pater familias, que dans celui de toute la civitas. L’instauration de la protection prétorienne 
pour des personnes avec lesquelles ils négocient sans qu'ils aient le commercium et sans l'engagement de la responsabilité du maitre, respectivement de leur pater familias pour de tels contrats, est sans conteste l'expression de cette tendance. C'est là que se manifeste l'importance des relations au sein des échanges civils et la mise en place d'une responsabilité illimitée ou limitée les concernant. C'est ainsi que s'équilibrent les intérêts des deux parties dans les rapports juridiques - l'intérêt du maitre, respectivement du pater familias pour les résultats de l'activité économique de ses esclaves et de ses alieni iuris, et la garantie des intérêts de tous, engagés de négocier avec ces personnes, avec l'assurance en plus que les engagements pris et les éventuelles indemnités seront accomplis.

Le thème de l'origine et de la nature des actiones adiecticiae qualitatis est directement lié à ces rapports. Dans la présente étude le thème sera traité vu la place de l'actio de peculio parmi les autres actiones adiectae 5 . L'attention sera fixée sur l'apparition et l'application de l'idée de la limitation de la responsabilité au niveau du montant d'un bien, déterminé et conçu comme un pécule, ce qui est une étape plus élevée de la limitation de la responsabilité en comparaison avec le cadre d'un ordre déterminé (chez l'actio quod iussu) ou en cas d'un enrichissement injuste réalisé (chez l'actio de in rem verso). Bien que les trois actions aient leur place dans le développement de la théorie sur la responsabilité limitée et la tradition

5 Voir mon étude « Actiones adiecticiae qualitatis » (en bulgare) . - In : Problèmes actuels du droit privé. Recueil des articles de la Conférence nationale en honneur de 95 anniversaire de prof. Emil Guéorguiev, arganisée par la Chaire des sciences civilistes de la Faculté de droit de l’Université de Sofia « Sv. Kliment Ohridski », Sofia, 2018, p. 64-92. et la bibliographie.

\section{7}


romaine en ce sens-là, l'actio de peculio se rapproche le plus à la responsabilité limitée des sociétés commerciales contemporaines ${ }^{6}$.

\section{La notion de pécule comme pratique et réglementation juridique}

\section{A. La terminologie et l'origine.}

L'étude du pécule comme pratique réglementée par l'Édit prétorien, dépassant entièrement le cadre du droit civil en matière d'incapacité pécuniaire des alieni iuris et incapacité civile des esclaves et débute ordinairement avec certaines précisions terminologiques.

Il est généralement admis que la notion "peculium" vient de "pecus" qui au début désignait le bétail en terme général, mais aussi vient du terme très ancien de "pecunia"7 signifiant des biens en bétail et en terres arables, et plus tard incluant l'argent ${ }^{8}$. Certains auteurs soulignent que "peculium" est le

\footnotetext{
${ }^{6}$ Вж. общо по темата PETRUCCI, A. Idee "vecchie" e "nuove" sulle attività imprenditoriali gestite all'interno di un peculio. -In : BIDR 106 (2012), p. 289-330.

${ }^{7}$ Pour la notion originaire de pecunia voir ANDREEV. M. Les notions "familia" et "pecunia" dans les textes des XII Tables. - In: Acta antiqua Philippopolitana. Vol. 1: Studia historica et philologica. Sofia,1963. p.173 ss. ; SMIRINE, V. La familia romaine et les représentations des romains concernant la proprieté. - In. L'homme et l'histoire. Recherches historicoanthropologiques et historico- culturelles). Moskva, 1990, p. 55-80., FEUVRIER-PREVOTAT, C. Le concept de la familia pecuniaque dans la Loi des XII Tables. - In : La question agraire a Rome : droit romain et societé. Perceptions historiques et historiographiques. Como, 1999, p. 59- 79 ; AMUNATEGUI PERELLO, C.F. Problems concerning familia in Early Rome. - In: Roman legal tradition, 4 (2008), p. 37-45 et la littérature récente.

${ }^{8}$ C'est la thèse principale dans les mauels cités en n.4.
}

678 
diminutif de "pecu", en développant plus tard la thèse qu'initialement le pécule consistait en petit bétail - des chèvres et des brebis - dont témoignent les auteurs anciens9. Ainsi par exemple Varro (cependant deux siècles après l'apparition du pécule comme il est généralement admis) mentionne que l'on donne aux esclaves du bétail pour le pâtre et pour s'entretenir de cette manière ${ }^{10}$. C'est Festus qui traite plus en détail l'étymologie et la nature du pécule comme étant lié au bétail (pecus), puisqu'il représente la richesse du pater familias ${ }^{11}$. Ces témoignages orientent évidemment vers une période plus ancienne, lorsque la question de la valeur du pécule ne se pose pas ou lorsqu'on est loin d'une pratique enfreignant et modifiant les principes fondamentaux dans la famille romaine et dans le traitement des esclaves avant et autour de l'époque des Guerres Puniques. Certains objets (mobiles surtout, mais parfois aussi immobiliers) sont donnés pour les administrer, éventuellement pour un usufruit, sans que ceci conduise à un changement dans la propriété du dominus/ pater familias.

\footnotetext{
${ }^{9}$ Voir en générale ainsi que la critique de ce concept GNOLI. F. Di una recente ipotesi sui rapporti tra "pecus". "pecunia". "peculium". - In: SDHI. Vol. 44. 1978. p. 204-218; BUTI. I. Studi sulla capacità patrimoniale.... cit. p. 19 ss.

${ }^{10}$ Varro. De re rust.. 1.2.17 “[...] agrius. tu. inquit. tibicen non solum adimis domino pecus. sed etiam servis peculium. quibus domini dant. ut pascant [...].

${ }^{11}$ Festus (De verb. sign.. v. peculium) Peculium servorum a pecore dictum est. ut est pecunia patrum familiae. En même sens voir aussi Varro (De lingua latina. (5.9.5) Pecus a quo pecunia inversa. quod in pecore pecunia tum consitebat pastoribus
} 
Certains auteurs considèrent que le texte d'Ulpianus fait écho de cette forme génétique d'affectation des biens de moindre valeur (petit bétail ou autres objets mobiles) et respectivement on use le diminutif:

D. 15.1.5.3. (Ulpianus libro 29 ad edictum) Peculium dictum est quasi pusilla pecunia sive patrimonium pusillum.

Les juristes romains se penchent rarement sur l'étymologie et s'ils le font c'est dans un but précis. En considérant les cas qui témoignent d'une large activité à plusieurs niveaux et très intense avec les biens de pécule où l'on applique l'actio de peculio (D.15.1. De peculio), on se rend compte que dans la période classique le pécule n'avait pas gardé son caractère initial de bien insignifiante ou de moindre somme. C'est encore plus évident en cas de actio tributoria (D.14.4.) s'il y a insolvabilité de l'esclave administrant des capitaux considérables. Il est à supposer qu'en incluant une telle constatation dans son commentaire sur l'Édit prétorien Ulpianus (un des juristes romains les plus précis quant à la terminologie et aux définitions) rappelle de façon rétrospective la genèse du terme (peculium dictum est), pour le différencier du pécule dans les nouvelles conditions économiques au-delà de la République tardive quand on ne parle plus de moindre montant de biens péculiaires. Les sources offrent des témoignages sur une activité très large avec la participation d'esclaves de différents maitres avec pécule, associés pour une activité commune (socios - D. 9.4.10), ou différents esclaves avec leurs pécules qui ménagent diverses activités de leur maître (D. 15. 1. 1. 6), y compris avec les pécules de vicarii, ce qui conduit à des liens horizontaux et verticaux des entreprises péculiaires, traitées comme des filiales indépendantes vis-à-vis de l'entreprise de base du maitre et représentantes 
dans leur ensemble ses formes de participation dans les rapports commerciaux ${ }^{12}$.

En désignant le pécule par le terme «patrimonium » Ulpianus met l'accent, de toute évidence, sur la conception de l'appartenance de ces biens au maître. Dès la République tardive le terme ne couvre plus seulement des res, mais englobe toute sorte d'appartenance de biens, y compris des droits et des obligations ${ }^{13}$. En séparant le patrimonium de pecunia, le juriste souligne d'une part que le pécule inclut aussi l'argent - soit initialement donné comme pécule pour des buts fixés par le maitre, soit comme résultat des transformations de biens péculiaires surtout dans les cas de libera administratio peculii ${ }^{14}$. Dans le droit classique la notion du patrimonium est également liée au montant de la valeur du pécule, montant avec lequel il figure dans le patrimoine du dominus / pater familias. Dès le II s. av. J.-C. on peut supposer que l'octroi de bétail ou d'autres objets en petite quantité comme

${ }^{12}$ L'étude des modèles d'organisation de l'activité économique des esclaves et des alieni iuris doit être effectuée séparément. On le mentionne seulement en relation du système complexe de coordination de la responsabilité limitée ou illimitée du dominus/ pater familias pour des contrats conclus par ses esclaves ou alieni iuris. Voir notamment SUÁREZ BLÁZQUEZ. G. Ocupación e intervención judicial de la empresa y de los holding de empresas peculiares comerciales de responsabilidad limitada, en suspensión de pagos, en la roma clásica. - In : Estudios de derecho, Vol. 71. №. 158 ( 2014), p. 675 ss.. който използва такава терминология. за да представи аналогичните на съвременните структури разрешения на римската юриспруденция. Voir aussi les textes y cités pour sa thèse: D. 15. 1. 1. 6; D. 15. 1. 7 . 4; D. 15. 1. 6; D. 15. 1. 17. ;D. 15. 1. 19; IGai. 4.73-74 et la littérature concernante des autres romanistes.

13 Pour la notion romaine du patrimonium voir LEPRI, M.F. Saggi sulla terminologia e sulla nozione del patrimonio in diritto romano,. Vol. I, Firenze, 1942; COLLINET, P., Bona et patrimoine : Les deux conceptions du patrimoine en Droit Romain, in: Etudes dédiées à la Mémoire d'André Andéadis , Athenes 1940, p.375-405; ANDREAU J., Patrimoines, échanges et prêts d'argent : l'économie romaine, Roma 1997. Срв.и HANISCH ESPINDOLA, H. El patrimonio en derecho romano. - In : Revista chilena de derecho, 4, No 1-6 (1977), p. 11-92 .

14 Cette clause donne la possibilité opérative la plus large avec les bines péculiaires - voir en générale la monographie de PESARESI, R. Ricerche sul peculium imprenditoriale. Bari, 2008. 
pécule n'est plus une hypothèse en masse, bien que cette pratique subsiste encore.

Dans les recherches sur le pécule en évolution, il est souligné que même si initialement on acceptait vers le III-II s. av. J.-C. l'octroi de bien insignifiant, l'emploi exact des termes et les notions de "pecus", "pecunia" et "peculium" reste discutable ${ }^{15}$. Cependant ce qui est certain c'est que l'économie romaine se développe et change son profil agraire vers l'économie de marché, et le pécule englobe déjà toute sorte d'objets, de l'argent, des créances, et que l'on admet toute transformation avec eux, de sorte que la pratique s'éloigne sensiblement de la notion initiale qui désignait ce terme.

\section{B. Les sources}

Le pécule dans sa forme initiale liée à l'esclavage et à l'économie agraire primaire est issu des témoignages dans les sources littéraires. Il est admis que les plus anciens nous viennent des comédies de Plautus vers la fin du IIIe s. av. J.-C., qui le mentionne comme pratique habituelle et courante dans 13 des 21 pièces qui nous sont parvenues. Le pécule est donné tant aux esclaves qu'aux fils, épouses, filles et revêt un caractère très varié, vu les objets qu'il englobe. Dans les comédies de Terentius du IIe s. av. J.-C. il est également mentionné bien qu'assez rarement dans des rapports relatifs au pécule.

La pratique y compris son évolution à partir de l'époque archaïque est expressément notée par Cato et Varro dans leurs traités sur l'agriculture et sur la mise en place d'une économie rationnalisée et orientée vers le marché ${ }^{16}$. La

\footnotetext{
15 Voir ZEBER. Op.cit., p. 9-10.

${ }^{16}$ Voir les témoignages de Varro et Festus pour le bétail en pécule : Varro, De re rust., 1.2.17 ... agrius, tu, inquit, tibicen non solum adimis domino pecus, sed etiam servis peculium, quibus
}

682 
bonne pratique du pécule, l'épargne et le travail, les bons comptes étaient de toute évidence un modèle de comportement au temps des Guerres puniques, ce qui est aussi reflété dans les sources littéraires ${ }^{17}$. Ainsi dans un texte de Ulpianus (D.11.3.1.5.), sont énumérés les cas dans lesquels un esclave, sous l'influence d'autrui, devient « méchant » et « enclin de mettre en désordre son pécule ». Ces normes morales ne dataient sûrement pas du temps d' Ulpianus, l'esclavage étant déjà en déclin ${ }^{18}$.

L'affirmation que le pécule existait au temps de la Loi des Douze tables (au milieu du Ve s. av.J.-C.) ou qu'il était réglementé en droit civil est aussi discutable. L'unique témoignage le concernant dans la Table VII.12, reconstruit selon les Règles d'Ulpianus (Ulpianus libro singularis regularum) stipule que si dans son testament le testateur avait disposé qu'un esclave devrait être libéré sous condition de verser à l'héritier 10 ooo, bien que l'esclave soit étranger à l'héritier, il devrait tout de même être libéré en payant à son acheteur ${ }^{19}$. Dans ce cas il s'agit en pratique de «rachat » de la liberté de l'esclave qui pouvait visiblement accumuler cette somme considérable en se servant de son pécule, et ceci dans les conditions d'échanges économiques actifs caractérisant la République tardive, mais difficilement admis pour le milieu du V s. avant J.Ch.(quand la Loi sur les

domini dant, ut pascant ; Fest., De verb. sign., v. peculium: Peculium servorum a pecore dictum est, ut est pecunia patrum familiae. 3 Pour le bétail comme la richesse des père des famille en époque archaïque voir aussi Varro, De lingua latina, 5.9.5. Pecus a quo pecunia inversa, quod in pecore pecunia tum consitebat pastoribus.

17 Pour les pécules dans la littérature latine voir ZEBER. Op. cit., p. 13 ss.

18 Plautus donne les preuves de pécule résultant de l'épargne des esclaves et des fils de faille en Plaut., Stichus. v. 60; Terent., Phormio. v. 43; Petron., Satir., v. 75.

19 Voir Lex XII Tabularum, Tab. VII.2. Sub hac conditione liber esse iussus si decem millia heredi dederit. etsi ab herede abalienatus sit. emptori dando pecuniam ad libertatem pervenient idque lex xii tabularum iubet . 
Douze tables a été promulguée) et lorsque le pécule comprenait un petit troupeau de bétail qui assurait la nourriture à l'esclave et à sa famille.

Les principaux témoignages sur la réglementation juridique du pécule sont contenus dans les textes des Digestes, en partie dans les institutions de Gaius et de Justinien et dans le Code de Justinien ${ }^{20}$. Bien qu'ils permettent de dégager certaines caractéristiques de l'institut juridique, ils reflètent dans l'ensemble le régime dans l'Édit prétorien, supposé être promulgué vers le Ier s. avant J.Ch., puisque l'Édit était utilisé en masse pour la participation des esclaves et des alieni iuris comme des représentants particuliers du dominus, respectivement du pater familias dans les échanges commercialles. Ceci ne modifie pas, mais au contraire, enrichit et développe le caractère initialement agraire de la pratique et crée une caractéristique généralisée du pécule, dans laquelle l'établissement du pécule signifie l'établissement de la responsabilité du propriétaire des biens (dominus, respectivement pater familias) pour les actions des personnes auxquelles il l'avait donné et qui sont juridiquement dépendants de lui.

Il est admis que le pécule passe dans son évolution par trois étapes:

- ancienne - dans laquelle le pécule est le moyen d'entretenir les esclaves et les alieni iuris - jusqu' à la fin du III s. et le début du IIe s. av. J.-Ch.,

\footnotetext{
${ }^{20}$ Le régime est concentré en D.15.1-2 ; D. 14.4. (en parallèle avec actio tributoria). On trouve certaines généralisations en IGai.4.69-74a et en IJ.4.7 pour les actiones adiectitiae qualitatis. En Code de Justinien on voit les trois actions assemblées dans un titre : CJ 4. 26. Quod cum eo qui in aliena est potestate negotium gestum esse dicetur. vel de peculio seu quod iussu aut de in rem verso. Mais il y a beaucoup des autres textes dispersés dans la Codification de Justinien par rapport des autres instituts. En somme voir le vocabulaire de Dydynskii (en russe : Дыдынский Ф.М.. Латинско-русский словарь к источникам римского права. Варшава, 1896 en https://lawbook.online/pravo-rimskoe/latinsko-russkiy-slovar-istochnikamrimskogo.html ).
} 
- réelle - du II s. av. J.-C. jusqu'à la fin du Ie s. ap. J.-C.- dans laquelle le pécule est un moyen commun et très répandu de participation des esclaves et des alieni iuris dans les contrats commerciaux en engageant la responsabilité limitée du propriétaire, respectivement du maître -,

- tardive - à partir du Ier s.- dans laquelle selon le modèle du pécule républicain, mais dans un but différent, sont réglementés les pécules spéciaux (peculium castrense et quasicastrense) des alieni iuris au service militaire, religieux et étatique, ce qui représente la voie vers leurs patrimoines indépendants et leur séparation sur le plan économique de la dépendance du pater familias.

Le commun pour les trois étapes est la sauvegarde de la nature du pécule comme bien particulier et indépendant séparé pour un but spécial et intuitu personae de l'esclave ou du fils de famille. C'est justement cette instauration qui constitue la condition indispensable à la responsabilité limitée du dominus/ pater familias en cas de contrats passés par des esclaves et des alieni iuris avec des biens péculiaires même dans la période la plus ancienne, car le pécule supposait une certaine indépendance dans son administration. Cependant, si vers le III-IIe s. av. J.-C. le pécule est encore traité comme la propriété du dominus/ pater familias, progressivement on note dans les Digestes son passage vers quasi proprium patrimonium filii/ servi. L'idée de son administration autonome selon la clause libera administratio peculii, se développe entièrement dans la création du régime des pécules spéciaux. Ainsi dans peculium castrense l'octroi de biens est lié au service militaire du fils de famille et ne crée aucune obligation pour pater familais, ce dernier n'intervenant pas de tout dans la façon d'administrer et

\section{5}


d'employer le pécule, n'assumant aucune responsabilité pour les contrats passés par le fils militaire, à l'exception des cas où il hérite son fils mort sans enfants et sans testament. Il en est de même du peculium quasicastrense. Dans ces deux cas l'emploi du terme peculium est par analogie en raison de la particularité et du statut d'un bien personnel protégé pour les fils de famille exerçant un service militaire, impérial et religieux (dans la période postclassique), et là ils assument la responsabilité jusqu'au montant de la valeur du bien péculiaire ${ }^{21}$.

\section{Actio de peculio comme une des actiones adjectitiae qualitatis}

Le régime juridique du pécule suit la voie typique du droit romain - à partir de la pratique établie on complète et amende le droit civil, selon lequel tout ce qui est acquis par les esclaves et par les alieni iuris du dominus/ pater familias et sa situation, peut être uniquement meilleur, et non pire $^{22}$. Les témoignages sauvegardés orientent vers les édits spéciaux, promulgués selon la plupart des auteurs vers le milieu ou plutôt vers la fin du IIe et le début du Ier s. av. J.-C., et régissant les prétentions pour une responsabilité du maître/ père de famille pour des contrats passés par ses esclaves ou les alieni iuris.

Ces actions, bien qu'appelées dans la théorie juridique avec le terme „actiones adiecticiae qualitatis“ - traduites littéralement comme « actions en

\footnotetext{
${ }^{21}$ Pour les pécules dits spéciales voir LA ROSA. F. I peculii speciali in diritto romano. Milano,. 1951 et la bibliographie précise.

22 C'est une règle- clé pour la compréhension des beaucoup des instituts de l'esclavage romain et c'est la raison d'être inclue dans le dernier titre du Digeste parmi les règles du droit ancien (De diversis regulis iuris antiqui): D. 50.17 .133 (Gaius libro octavo ad edictum provinciale) Melior condicio nostra per servos fieri potest. deterior fieri non potest.
}

686 
justice avec qualité ajoutée », ne représentent pas une catégorie unie, ni ont un régime commun ${ }^{23}$. Chacune d'elles a ses fondements spécifiques pour être catégorisée et c'est uniquement leur emplacement par les compilateurs de Justinien dans deux livres séparés des Digestes permet de tirer des certaines conclusions et faire la distinction entre la responsabilité limitée et la responsabilité illimitée du dominus/ pater familias les concernant ${ }^{24}$.

Selon la majorité des auteurs les actiones adiecticiae qualitatis représentent l'image même de la représentation directe, puisque selon le régime juridique la négociation avec des esclaves et des alieni iuris se fait au nom du pater familias et le résultat des contrats passent directement dans son patrimoine, la non-observation des engagements pris conduisant directement à sa responsabilité envers les partenaires ${ }^{25}$.

Talamanca considère pourtant que la représentation directe est réduite aux seuls cas dans lesquels le magister navis, respectivement institor est une personne libre, exerçant les droits du dominus (comme propriétaire du bien) sur la base de sa volonté unilatéralement exprimée, en déterminant

23 On ne trouve dans les sources aucune généralisation de ces actions que dans l'unique titre des Institutions de Justinien (IJ.4.7.) Il est admis que la notion des actiones adjecticiae qualitatis est introduit des Glossateurs- voir BONFANTE, P. Istituzioni di diritto romano, Milano, 1987, p.147.

24 Voir pour ces actions en générale MICELI. M. Sulla struttura formulare delle 'actiones adiecticiae qualitatis'. Torino. 2001.

25 Voir RICCOBONO, S. Lineamenti della dottrina della rappresentanza diretta in diritto romano. - In: AUPA. 14 (1930). p. 392 ss.; ID.. Corso di diritto romano. v. II. Milano. 19331934. p. 257 ss.; HAMZA. G. Aspetti della rappresentanza negoziale in Diritto romano. - In: INDEX 9 (1980). p. 193-229; MICELI. M. Studi sulla rappresentanza nel diritto romano. Milano. 2008. p. 1-8; ID. Sulla struttura formulare delle 'actiones adiecticiae qualitatis'.Torino. 2001 . p.185-224; WACKE.A. Alle origini della rappresentanza diretta: le azioni adiettizie. -In : Nozione formazione e interpretazione del diritto dall'età romana alle esperienze moderne. Melanges F. Gallo. v.I. Napoli. 1997. p. 583- 615 ; LIGIOS. M.A. Nomen negotiationis. Profili di continuita e di autonomia della negotiatio nell'esperienza giuridica romana. Torino. 2013.

\section{7}


ainsi les limites de leur pouvoir représentatif particulier ${ }^{26}$. Dans l'analyse des textes inclus dans D.14.1-4 et D.15.1-4, il y a en réalité des hypothèses dans lesquelles participent uniquement des personnes libres, mais ce ne sont pas les cas typiques. De toute évidence l'intervention prétorienne est due au fait que l'engagement des esclaves et des alieni iuris dans des opérations commerciales conduit à des rapports juridiques entre eux et leurs partenaires, et les désigner comme naturales obligationes n'offre point de garanties pour ces derniers en cas de non-exécution. Si on acceptait une représentation directe de la part d'esclaves et de membres de famille dépendants, même dans sa variation romaine, alors il n'y a pas de raison d'avoir des actions, puisque la responsabilité du dominus/ pater familias est sous-entendue et elle devrait toujours être in solidum ${ }^{27}$.

Ainsi dans l'impossibilité d'appliquer les critères actuels pour la représentation, traditionnellement (et avec une argumentation plus au moins détaillée) l'institut du pécule, ainsi que les autres instituts liés à la responsabilité adiectice, dans la plupart des cours universitaires sont examinés uniquement dans le contexte d'adoucir l’incapacité pécuniaire des esclaves et des alieni iuris au fond des particularités de l'esclavage et de l'organisation familiale des Romains ${ }^{28}$. Telle est aussi la position d'Albanese

${ }^{26}$ TALAMANCA. M. Istituzioni di diritto romano. Milano. 1990, p. 86.

27 Voir en ce sens BURDESE. A. Manuale di diritto privato romano. 3 ed.. Torino. 1975. p. 509. nt.1; MARRONE. M. Istituzioni di diritto romano. Milano. 1994. p.197.

${ }^{28}$ Dans le manuel de droit romain privé en bulgare (Andréev, M., Op. cit., $\mu$ on en parle pour la capacité patrimoniale des esclaves- p. 156-157 et des alieni iuris - p. 177-178. De meme est dans les autres cours de droit romain privé- voir par ex. en BETTI. E. Diritto romano. I. Parte generale. Padova. 1935. p. 128; ID.. Istituzioni di diritto romano. v. I.2. Padova. 1942. p. 60; LONGO. G.Manuale elementare di diritto romano. Torino. 1939. p. 113. p. 115 ss.; GARCÌA GARRIDO. M. J. Derecho privado romano. I. Instituciones. 2e ed.. Madrid. 1982. p. 454; PUGLIESE. G. Istituzioni di diritto romano. 3 ed. (con la collaborazione di F. Sitzia e L. Vacca). Torino. 1991. p. 252; ZIMMERMANN. R. The law of obligations. Roman foundations of the 
et il centre son analyse sur le statut personnel des personnes réalisant une activité commerciale et assumant une responsabilité dans le cadre d'actiones adiecticiae qualitatis. Pour lui l'essentiel est non le pouvoir exercé sur l'esclave, mais plutôt la propriété du bien qui lui est accordé pour l'administrer, qui est la raison pour la responsabilité du dominus. L'envergure des pouvoirs de l'esclave est graduée à partir de iussus à travers le peculium jusqu'au praepositio, qui se répercute sur le caractère de chacune des actions adiectices, considérées comme une catégorie historique par laquelle les esclaves et les alieni iuris acquièrent en fait l'administration de biens d'autrui. Le préteur légalise en réalité une capacité liée à l'administration réelle d'un bien d'autrui par les esclaves et les alieni iuris qui opèrent comme intermédiaires dans les rapports commerciaux entre le maître et les tiers personnes ${ }^{29}$.

En plus les actions sont associées aux différents modèles organisationnels de l'activité commerciale et entrepreneuse à Rome de la République tardive et au temps de Principat et leur distinction en fonction de la responsabilité limitée ou illimitée du dominus /pater familias envers des tierces personnes est commentée en prenant en considération le genre et l'envergure de l'activité des alieni iuris ${ }^{30}$. Elles sont examinées comme moyen

civilian traditions. Cape Town-Wetton - Johannesburg. 1990. rist. Oxford. 1996. p. 52 nt. 127; D’ORS. A. Derecho privado romano. 9 ed.. Pamplona. 1997. p.303; IGLESIAS. J. Derecho romano. 12 ed. . Barcellona. 1999. p. 246; Scherillo. G.. Gnoli. F. Diritto romano. Lezioni istituzionali. Milano. 2003. p. 110 etc. .

29 Voir ALBANESE. B. Le persone nel diritto privato romano. Palermo. 1979. p. 146-162.

30 Voir SUAREZ BLASQUEZ. G. Derecho de empresas en la Roma clasica. Madrid, 2014 ; MILLER. G.P. Economics of ancient law. Cheltenham. 2010. Voir la généralisation de ces problèmes en A. DI PORTO. Il diritto commerciale romano una 'zona d'ombra'nella storiografia romanistica e nelle riflessioni storico-comparative dei commercialisti. In: Nozione. formazione e interpretazione del diritto dall'età romana alle esperienze moderne. Ricerche dedicate al prof. F. Gallo. v. III. Napoli. 1997. p. 413 ss.. както и WACKE. A. Le azioni adiettizie. cit.. p. 585 ss.

\section{9}


de partage du risque, d'où la construction particulière en justice puisque les relations internes entre les esclaves et les alieni iuris d'une part et leur dominus/ pater familias ont de l'importance dans la mesure où la responsabilité de ce dernier est illimitée (avec actio exercitoria et actio institoria) ou limitée (avec actio de peculio, actio quod iussu, actio de in rem verso $)^{31}$. La distinction de cette responsabilité est examinée tant sur le plan historique que sur le plan économique, en considérant l'évolution et son existence parallèle vers la fin de la République, dans les conditions d'une expansion territoriale et économique de Rome, de contrats isolés ou similaires avec des pouvoirs limités aux activités pécuniaires de volume différent ou aux entreprises, production ou concession entière du commerce ordinaire ou maritime.

Dans ces conditions les projections sociales du pouvoir du maître sur les esclaves et du père sur les alieni iuris acquièrent d'autres dimensions juridiques, auxquelles le préteur ne peut point s'abstenir d'interférer. L'orientation de ces personnes vers une initiative économique se manifeste dans un contexte économique différent - faire participer une grande partie de la population apte au travail dans des différentes activités, en se servant de diverses constructions juridiques - mieux adaptées aux objectifs économiques correspondants $^{32}$. Ainsi par exemple Serrao considère que c'est bien l'opportunité économique qui est à la base des concepts et des formes juridiques des différentes entreprises commerciales, et la responsabilité de leurs propriétaires pour l'activité des personnes qui opèrent directement avec

${ }^{31}$ Voir BUCKLAND. W.W. , MCNAIR. A. D. Roman law and common law. 2 ed.. Cambridge. 1974. p.217-220.

$3^{2}$ Voir SERRAO, F. Impresa e responsabilità a Roma nell'età commerciale. Pisa. 2002. p.1764. 
les biens et les capitaux envers de tiers personnes est le résultat de l'appréciation du préteur de cette opportunité. Les principes de la responsabilité limitée ou illimitée sont fondés sur la logique économique, le droit la suit et lui donne une forme déterminée 33 .

Di Porto et Cerami développent la même thèse en présentant des entreprises individuelles ou collectives avec la participation des esclaves et des alieni iuris auxquelles peuvent se joindre les maîtres des esclaves, même des co- propriétaires des esclaves, et de créer des sociétés communes avec des esclaves pour administrer des biens communs. A la fin de la République la vie d'affaires à Rome offre un tableau si différent quant au statut des personnes qui y participent, qu'il est difficile de déterminer les formes juridiques typiques pour leur mise en œuvre. La nature économique et l'envergure de l'entreprise commerciale conditionnent la responsabilité limitée et illimitée pour les actions adiectices, qui sont non seulement une caution, mais aussi un stimulant pour une activité économique d'envergure, suffisamment garantie par elles 34 .

Dans le cadre de cette revue conceptuelle l'étude du pécule a sa place spécifique, car elle se situe dans une position intermédiaire entre une large activité commerciale à plusieurs niveaux de l' institor et l'exercitor d'une part, et une autre activité économique plus restreinte de par son volume, comprenant des contrats isolés ou similaires chez iusus ou d'un enrichissement réalisé sans une procuration spéciale (versio). Dans le titre Ier du livre XV des Digestes relatif à l'actio de peculio on voit une activité très

33 Voir SERRAO. Op. cit., p. 20-21.

34 Voir DI PORTO. A. Impresa collettiva e schiavo 'manager' in Roma antica (II sec. a.C.IIsec.d.C.). Milano. 1984. p.18-46; CERAMI. P.. DI PORTO. A.. PETRUCCI. A. Diritto commerciale romano. 2 ed.. cit.. p. 11-41. Срв. и AUBERT. J. J.Business managers in ancient Rome: a social and economic study of institores. 200 B.C.-A.D. 250. Leiden . 1994. p. 46-100.

\section{1}


large, sous différentes formes, à savoir beaucoup d'esclaves avec pécule appartenant à un ou à plusieurs maîtres, beaucoup de pécules appartenant à un esclave, participation dans une société avec des pécules, pécules avec copropriétaires - esclaves, présentation partielle du capital administré comme pécule, des pécules de vicaires etc. ${ }^{35}$ En cas d'insolvabilité et de partage des biens dans l'actio tributoria de ce qui est présenté comme pécule, on note dans $D$. 14.4. une très grande diversité et souvent une complexité dans les relations relatives aux biens, et ceci dans le cadre d'un même patrimoine, relations qui sont réalisées au moyen de pécules ou au moyen d'autres formes d'activité commerciale confiée aux esclaves et aux alieni iuris.

On ne trouve pas dans les sources des renseignements sur le choix des préférences, ce qui nous oriente vers des réflexions générales sur la responsabilité limitée, valables d'ailleurs dans le droit commercial contemporain aussi ${ }^{36}$. Quant à Rome et à son économie plus primitive du point de vue contemporain de telles conclusions ne sauraient être acceptées apriori. Dans le régime du pécule on observe une sensibilité particulière pour la comptabilité concernant le bien pécuniaire et pour la limitation de la responsabilité du propriétaire du pécule, la comparaison entre tierces personnes et les garanties pour leurs intérêts jusqu'au niveau de la valeur du pécule, c'est-à-dire du capital associé, la distribution du risque etc. Cependant il ne faut pas oublier que les juristes romains ne font pas de distinction entre le droit civil et le droit commercial et une même forme comme c'est le cas du

35 D.9.4.10; 14.4.11; 15.1.1.6.; 15.1.7.4; 15.1.6.; 15.1.17; 15.1.19 etc. .

36 Voir l'argumentation fondamentale en FEZZA. F. Profili organizzativi dell'impresa e disciplina della responsabilità nel diritto romano e nel diritto contemporaneo. - In : Teoria e $\begin{array}{llllll}\text { storia del } & \text { diritto } & \text { privato. } & 2 & \text { (2009) }\end{array}$ http://www.teoriaestoriadeldirittoprivato.com/index.php?com=statics\&option=index\&cID= 70

\section{2}


pécule peut signifier l'octroi de biens pour les besoins personnels des alieni iuris et des esclaves, mais aussi l'octroi des biens à de grandes entreprises de production et aux entreprises commerciales, lorsqu'on détermine les limites de la valeur des biens, y compris les apports y relatifs. Il y a en plus un autre trait particulier : l'activité commerciale est associée au statut des personnes qui opèrent avec des biens pécuniaires. En cas de cessation de la dépendance ou de décès du dépendant ou de l'esclave, ou bien du maitre, le pécule est sauvegardé et il garantit les créances des créanciers dans un délai d'un an suivant l'événement - c'est bien le sens du régime de l'actio de peculio annalis (D.15.2.). L'appréciation du choix du pécule face aux autres formes d'activité commerciale, sanctionnées par les actions adiectices peut être liée à la possibilité de contrôle effectif du propriétaire du bien sur l'activité. Le genre d'activité, la place de sa réalisation, les conditions économiques et politiques générales ont également leur importance, le contexte social et l'efficacité de la juridiction au moment donné comptent aussi.

C'est de ce point de vue que nous pouvons examiner l'évolution de la responsabilité adiectice et la place du pécule dans le système des actions prétoriennes. Il est généralement admis qu'elles ont été créées vers le IIe s. av. J.-C., mais il n'y a pas de raison d'accepter que toutes les six actions sont apparues en même temps, bien que, comme le note Guarino ${ }^{37}$, elles figurent dans deux titres différents dans les Digestes, mais sont inclues ensemble dans l'Edictum perpetuum, étant définitivement réglementées dans la période classique.

Ả la question fondamentale quelle est la responsabilité qui apparaît en premier - la limitée ou l'illimitée, les juristes romains ne donnent pas une

37 Voir GUARINO. A. Actiones adiecticiae qualitatis. In: NNDI . v. 1.1. Torino. 1957. p. 271.

\section{3}


réponse unique. Le pécule se manifeste comme pratique dès le III s. av. J.-C. et au début il est octroyé pour s'entretenir, stimulant l'intérêt des esclaves d'avoir un petit troupeau d'ovins ou autre moindre bien ${ }^{38}$. Probablement les esclaves, mais parfois aussi les alieni iuris recevaient des ordres pour des contrats uniques ou des contrats types que l'on pourrait nommer avec le terme général iussus ${ }^{39}$. Avec le développement progressif de ces pratiques devenues de plus en plus nombreuses pour des cas sans un ordre ou un bien expressément octroyé, mais où il y a un enrichissement injuste, on suppose la création de l'actio de in rem verso comme dérogation à la règle qui stipulait qu'à travers les esclaves la situation du maitre ne devrait que s'améliorer ${ }^{40}$.

On suppose logiquement que la responsabilité, fixée dans l'édit prétorien, fait partie au début d'un engagement plus limité du maitre/ père de famille, allant ensuite vers son engagement total pour son bien - objet des contrats passés par les esclaves et par les alieni iuris dans le cadre d'une activité commerciale beaucoup plus large et plus intense. Le point de vue de Gaius dans IGai.4.69 vient en appui à cette thèse, repris dans le paragraphe initial du titre VII du livre IV des Institution de Justinien, dans lequel on

${ }^{8}$ On le voit clairement dans le texte de Varro qui décrive une pratique assez ancienne : Varro. De re rust.. 1.2.17 "[...] agrius. tu. inquit. tibicen non solum adimis domino pecus. sed etiam servis peculium. quibus domini dant. ut pascant [...].

39 Sur le thème voir en générale COPPOLA. G. Lo iussum e la sostituzione negoziale nell'esperienza romana. Milano. 2003 avec une riche bibliographie. и цитираната там литература. Il y a des hypothèses que même actio quod iussu précède actio de peculio dans son modèle de limiter la responsabilité selon l'activité préposée et les biens conférés pavec un iussum au avec une clayse plus libérale de disposition dans le cas de pécule. Voir en ce sens ALBANESE. B. Le persone nel diritto privato romano. cit.. p.16o ss. qui accepte actio quod iussu comme plus ancienne. Voir aussi la discussion chez CERAMI. P. . DI PORTO. A.. PETRUCCI. A. Lezioni di diritto commerciale romano. Op.cit., p.45.

$4^{\circ}$ Pour l'apparition tardive de l'actio de in rem verso voir MACCORMACK, G. The later history of the "actio de in rem verso" (Proculus- Ulpian). -In: SDHI. 48 (1982). p. 318- 367.

\section{4}


commence par le régime de l'actio de peculio ${ }^{41}$. Le titre est consacré à ceux qui sont dépendants et passent des contrats (Quod cum eo qui in aliena potestate est negotium gestum esse dicitur) et on y examine progressivement l'actio quod iussu, l'actio exercitoria, l'actio insitoria comme des actions du même type, engageant la responsabilité illimitée du pater familias/ dominus, et l'actio tributoria, l'actio de peculio, l'actio de in rem verso comme des actions à responsabilité limitée jusqu'à un certain montant ${ }^{42}$.

Il me semble que la systématique dans les Institutions, tout aussi dans les Digestes (D.14.1-4; D.15.1-4) et dans le Code de Justinien (CJ 4. 25. De exercitoria et institoria actione и СЈ 4. 26 . Quod cum eo qui in aliena est potestate negotium gestum esse dicetur, vel de peculio seu quod iussu aut de in rem verso) ne répond pas à la question sur l'ordre d'apparition du régime. On suit plutôt le concept sur la distinction entre la responsabilité limitée et illimitée, l'accent étant mis sur cette dernière comme une préoccupation plus radicale et intense pour les intérêts des créanciers dans des contrats passés avec des alieni iuris et des esclaves. Au VI s. au cours de la compilation des Digestes on utilise une voie logique en partant du commun vers le privé, ce qui explique peut-être cette systématique.

41 IJ.4.7.pr. Quia tamen superius mentionem habuimus de actione quae in peculium filiorumfamilias servorumque agitur: ... On traite l'actio de peculio dans le même titre sous l'aspect des conditions de procédure : IJ.4.7.4. Praeterea introducta est actio de peculio deque eo. quod in rem domini versum erit. ut. quamvis sine voluntate domini negotium gestum erit. tamen sive quid in rem eius versum fuerit. id totum praestare debeat. sive quid non sit in rem eius versum. id eatenus praestare debeat quatenus peculium patitur.

${ }^{42}$ Chez les glossateurs il y a une systématisation différente mais sans donner des raisons de ce rangement des actions. On suppose comme critère les limites de la responsabilité. Voir ACCVRSII. Instititutes Iustiniani Augusi. Venetiis. 1574. p.714. cité par P. CERAMI. DI PORTO. A.. PETRUCCI. A. Lezioni di diritto commerciale romano. Op.cit., p. 45 ss..

\section{5}


On peut également admettre que l'on a suivi le régime final des actions dans Edictum perpetuum, sans prendre en considération sa genèse et son évolution. Il est pourtant indiscutable que dans l'un des édits tout ce qui concernait le pécule avait été uni à un moment dans l'actio de in rem verso et actio quod iussu, comme l'affirme Ulpianus:

D. 15.1.1 (Ulpianus libro 29 ad edictum) pr. Ordinarium praetor arbitratus est prius eos contractus exponere eorum qui alienae potestati subiecti sunt, qui in solidum tribuunt actionem, sic deinde ad hunc pervenire, ubi de peculio datur actio.

1. Est autem triplex hoc edictum: aut enim de peculio aut de in rem verso aut quod iussu hinc oritur actio.

Indépendamment des théories existant sur cette questions, il faudrait prendre en compte le fait que les commentaires de Paulus et de Ulpianus généralement inclus dans les Digestes, ainsi que la systématisation de cette matière dans l'Edictum perpetuum datent de presque deux siècles après la promulgation de l'édit, respectivement des édits des préteurs concernant ces actions, déterminées comme indiscutablement utiles ${ }^{43}$. C'est cette même logique que les compilateurs des Institutions et du Code du VI s. ont suivi en groupant toutes les actions adiectices dans un titre, puisque dans cette période l'esclavage et les relations familiales sont radicalement différents, avec une autonomie des biens considérable des alieni iuris de ceux de la fin de la République, et le partage du risque et de la responsabilité correspond à la représentation et à l'hypothèse plus typique pour une négociation de personnes libres.

43 Voir D. 14.1.1. pr. (Ulpianus libro 28 ad edictum) Utilitatem huius edicti patere nemo est qui ignoret .... 
Or, on ne peut ne pas faire une comparaison des hypothèses concernant l'emploi de l'actio tributoria et la déduction du pécule examinées dans D.14.4. Ulpianus considère qu'en présence de pécule dominus/ pater familias a le privilège particulier d'exclure le bien pécuniaire (merx peculiaris) de la masse commune de l'insolvabilité. Même si le pécule est établi expressément ou tacitement, il est toujours nécessaire d'avoir une pleine certitude sur son existence :

D.14.4.1 (Ulpianus libro 29 ad edictum) pr. Huius quoque edicti non minima utilitas est, ut dominus, qui alioquin in servi contractibus privilegium habet (quippe cum de peculio dumtaxat teneatur, cuius peculii aestimatio deducto quod domino debetur fit), tamen, si scierit servum peculiari merce negotiari, velut extraneus creditor ex hoc edicto in tributum vocatur. ... 2. Peculiarem autem mercem non sic uti peculium accipimus, quippe peculium deducto quod debetur accipitur, merx peculiaris, etiamsi nihil sit in peculio, dominum tributoria obligat, ita demum si sciente eo negotiabitur.

Par conséquent toutes les actions adiectices, n'importe leur création dans le temps, sont déterminées comme particulièrement nécessaires et utiles, tandis que dans les titres correspondants des livres XIV et XV des Digestes, tout comme dans les autres sources, on constate leur application généralisée avec des hypothèses complexes dont se sert le préteur pour les identifier et donner, ainsi que le mixage compliqué de formes organisationnelles pour les relations commerciales avec l'inclusion des alieni iuris, tout ceci suscitant une riche casuistique. Même la tentative de généraliser et de typer que l'on trouve dans les Institutions et dans le Code de Justinien, offre une diversité exceptionnelle de potentialités pour mettre en œuvre tout le potentiel d'affaires des romains dans les conditions d'un État important de par son territoire et ses possibilités économiques. 


\section{Détermination et séparation patrimoniale du pécule}

\section{A. Définition du pécule.}

Le pécule est ordinairement déterminé comme un "bien séparé », octroyé par le pater familias ${ }^{44}$ pour être administré et géré par les fils de famille et les esclaves. Les différents auteurs mettent l'accent sur les divers aspects de l'institut, mais on souligne dans l'ensemble que c'est avec ce bien que les fils de famille et les esclaves participent dans la vie économique de l'ancienne Rome au nom de son pater familias / dominus.

Le point de vue d'Ulpianus est cité en appui et se réfère à des renseignements fournis par Celsus qui lui cite une définition de Tubero45:

D.15.1.5.4. (Ulpianus libro 29 ad edictum) Peculium autem tubero quidem sic definit, ut celsus libro sexto digestorum rifert, quod servus domini permissu separatum a rationibus dominis habet deducto unde si quid domino debetur.

$\mathrm{Vu}$ le temps où vivent les trois juristes, on peut admettre que cette définition de Tubero est suffisamment authentique, puisqu'elle est créée vers la fin de la République quand probablement a été promulgué l'édit introduisant l'actio de peculio, qui de sa part, reflète une pratique établie et la nécessité d'un régime et d'une protection des parties lors de la négociation avec un bien pécuniaire dès la fin du II s. et le début du Ier s. av. J.-C. La

\footnotetext{
44 Voir ANDRÉEV. Op.cit., p. 157-8. p.177-8; CUQ, E. Manuel des institutions juridiques des Romaines. P.1928. p. 143 ss. ; GIRARD, P. Op.cit., p.170 s.; TALAMANCA. Op. cit.. p. 77, p. 122 ss.; NUOVO DIZIONARIO GIURIDICO ROMANO. Napoli, 1998, p. 405-406.

45 Quintus Aelius Tubero est un jurisconsulte et homme politique romain de l'époque augustéenne et il présente la notion du pécule établie vers la fin de la République dans la pratique et selon l'Edit du préteur urbain.
} 
notion de pécule est tout à fait connue au début du II s. quand vit Celsus et elle est pleinement et indiscutablement admise par Ulpianus au III s. ${ }^{46}$

À cette définition s'ajoute comme commentaire au droit civil un fragment de Pomponius du milieu du II s. :

D.15.1.4.pr. (Pomponius libro 5 ad Sabinum) Peculii est non id cuius servus seorsum a domino rationem habuerit, sed quod dominus ipse separaverit suam a servi rationem discernens.

Traditionnellement ces textes sont admis comme définition du pécule et interprétés dans le sens que le pécule ne comprend pas seulement des objets concrets le composant, mais il compte en raison de sa valeur et s'approche en quelque sorte de la notion générale du capital, faisant partie du patrimoine du pater familias. L'analyse des opinions de la jurisprudence romaine, figurant dans le livre XV, titre 1 des Digeste, nous fait réfléchir sur ce que représente le pécule vers le II-III s.

\section{B. Séparation de comptes}

Ces définitions sont très souvent interprétées dans un sens très étroit et limité, uniquement sur le plan de la comptabilité. Initialement le pécule était conçu par sa caractéristique réelle sur laquelle il y a dans les Digestes pas mal de textes qui soulignent que le pécule peut englober toute sorte d'objets meubles y compris vêtements ${ }^{47}$, argent, bétail, mais aussi des terres et des

${ }^{46}$ Pour la définition du pécule voir AMIRANTE, L. Lavoro di giuristi sul peculio. Le definizione da Q. Mucio a Ulpiano. - In : Studi in onore di Cesare Sanfilippo. Vol. 3. Milano. p. 5 ss. Pour l'hypothèse selon laquelle Masurius Sabinus est le vrai auteur de la définition voir DI PORTO, A. Impresa collettiva e schiavo manager in Roma antica. Milano, 1984, p.288, n. 60.

47 Voir le cas commenté pa Poponius où des vêtements peuvent être fournis en tant que peculium, mais pour usage permanent. E non en cas occasionnel ou avec un but prévu : D.15.1.25 (Pomponius libro 23 ad Sabinum) Id vestimentum peculii esse incipit. quod ita 
bâtiments $4^{4}$, et même des esclaves (vicarii) et leurs pécules ${ }^{49}$. Ulpianus généralise en notant que le pécule peut comprendre toute sorte d'objets meubles et immeubles, des créances ${ }^{50}$, héritages, légats, universitas :

D.15.1.7 (Ulpianus libro 29 ad edictum) 2. Scire autem non utique singulas res debet, sed paxumeresteron [id est : magis in universum], et in hanc sententiam Pomponius inclinat.

4. In peculio autem res esse possunt omnes et mobiles et soli : vicarios quoque in peculium potest habere et vicariorum peculium : hoc amplius et nomina debitorum.

5. Sed et si quid furti actione servo deberetur vel alia actione, in peculium computabitur : hereditas quoque et legatum, ut Labeo ait.

Bien que rarement on rencontre dans les sources le terme "res peculiares" 51 , et plus souvent merx peculiaris, qui a un sens spécifique que nous aborderons infra.

Cependant il y a lieu de souligner que certains auteurs examinent comme objets du pécule les droits et les obligations des parties,

dederit dominus. ut eo vestitu servum perpetuo uti vellet eoque nomine ei traderet. ne quis alius eo uteretur idque ab eo eius usus gratia custodiretur. Sed quod vestimentum servo dominus ita dedit utendum. ut non semper. sed ad certum usum certis temporibus eo uteretur. veluti cum sequeretur eum sive cenanti ministravit. id vestimentum non esse peculii.

48 Pour les imeubles dans le pécule voir D.15.1.7.4. D.15.1.22- 23 et expressément en D.33.8.6.pr. (Ulpianus libro 25 ad Sabinum ) Si peculium legetur et sit in corporibus. puta fundi vel aedes...Pour un légat de fundus instructus cum servi comme pécule voir D. 15.1.54 (Scaevola libro primo responsorum) Filio familias uni ex heredibus praedia praelegavit ut instructa erant cum servis...

49 Pour les objets de pécule voir le chapitre III de mon livre Peculium. Caractérstique objective et subjective. Sofia, 200o. Pour le vicariat voir REDUZZI MEROLA, F. Servo parere. Studi sulla condizione giuridica degli schiavi vicari e dei sottoposti a schiavi nelle esperienze greca e romana. Napoli, 1990,. p. 2-19; p. 23 ss.

$5^{0}$ Voir pour les obligations dans active du pécule chez MICOLIER. Op.cit., p. $163 \mathrm{~s}$.

${ }^{51}$ Voir D. 15.1.9.1. ; D. 6.1.56; D .18.1.40.5. On parle de l'usage des res peculiares en. D.33.6.9.3. 
respectivement leurs possibilités juridiques d'opérer avec les objets et les droits, inclus dans le pécule et non les objets mêmes ${ }^{52}$. Les renseignements dans les Digestes montrent de façon différenciée les possibles objets de l'octroi pécuniaire, qui probablement sont appréciés et évalués comme pécule.

Ulpianus, lui-même, dans D.15.1.5.3 souligne justement la caractéristique réelle du pécule. Ce texte précise que le pécule ne comprend pas seulement l'argent, mais l'ensemble d'objets (y compris dans le sens d'universitas) et de droits, le terme étant utilisé aussi pour le patrimonium ${ }^{53}$. Ceci a trait d'une part, au statut autonome qu'il acquiert dans la période classique, et de l'autre, à la possibilité d'opérer librement avec lui comme avec tout autre bien de personnes ayant droits, et ceci dans l'observation des restrictions fixées. Dans le développement de cette thèse certains auteurs cherchent l'instauration du pécule comme un quasi- patrimoine autonome, pareil à l'hereditas (y compris dans l'hypothèse de l'hereditas iacens) ou de personne morale dans le sens moderne de cette notion ${ }^{54}$.

Les Postglossateurs se penchent sur des exemples, dans lesquels le pécule comprend tant des droits réels (res, fructus), que des obligations (usura) 55. La mention de pecunia comme objet du pécule est dans le sens

$5^{2}$ В този смисъл вж. BUCKLAND . op.cit.. p. 188 s.; Hervieux . op.cit. p. 19 s.

53 Pour la notion du patrimonium voir LEPRI, M.F. Saggi sulla terminologia e sulla nozione del patrimonio in diritto romano. Firenze 1942; COLLINET, P. Bona et patrimoine : Les deux conceptions du patrimoine en Droit Romain. in: Etudes dédiées à la Mémoire d'André M. Andréadès. Athenes, 1940. p.375-405; ANDREAU, J. Patrimoines. Échanges et prêts d'argent : l'économie romaine. Roma, 1997; BARREIRO, A.F. , PARICIO J.. Fundamentos de derecho patrimonial romano. Madrid, 1998.

54 Вж. SUÁREZ BLÁZQUEZ. G. El peculio como ente jurídico autónomo y matriz de la "merx peculiaris". - In: Revista de Estudios Histórico-Jurídicos. 32 (2010). p. 119- 125 et la bibliographie y citée.

55 On utilise le mot comme l'usage, mais ici c'est le sens juridique des intérêts comme des friuts civiles- c'est tout à fait logique de les faire partie du pécule. 
d'argent, mais aussi dans le sens de bien (comme synonyme du patrimonium, la notion étant étroitement liée au statut de pater familias).

En examinant les objets du pécule, on ne peut ne pas remarquer le déploiement du système des pécules aux servi vicarii et nombre de textes liés aux vicarii notent la possibilité de créer une multitude de sub-pécules, qui sont concernés par la responsabilité limitée ils aussi :

D.15.1.17 (Ulpianus libro 29 ad edictum) Si servus meus ordinarius vicarios habeat, id quod vicarii mihi debent an deducam ex peculio servi ordinarii? Et prima illa quaestio est, an haec peculia in peculio servi ordinarii computentur. Et Proculus et Atilicinus existimant, sicut ipsi vicarii sunt in peculio, ita etiam pec se penchent sur ulia eorum: et id quidem, quod mihi dominus eorum, id est ordinarius servus debet, etiam ex peculio eorum detrahetur: id vero quod ipsi vicarii debent, dumtaxat ex ipsorum peculio: sed et si quid non mihi, sed ordinario servo debent, deducetur de peculio eorum quasi conservo debitum: id vero, quod ipsis debet ordinarius servus, non deducetur de peculio ordinarii servi, quia peculium eorum in peculio ipsius est (et ita servius respondit), sed peculium eorum augebitur, ut opinor, quemadmodum si dominus servo suo debeat..$^{6}$

Tout le régime du pécule dans les Digestes devrait être examiné en tenant compte du développement historique de l'institut. Indépendamment du fait que ce sont les opinions des juristes classiques de la fin du II et du III s. qui prévalent, elles contiennent, elles aussi, des réminiscences de précédentes époques. C'est dans ce sens que devraient être interprétés les renseignements concernant la caractéristique objective du bien composant le pécule.

\footnotetext{
${ }^{6}$ Voir D. 15.1.6 (Celsus libro sexto digestorum) Definitio peculii quam Tubero exposuit ut Labeo ait. ad vicariorum peculia non pertinet. quod falsum est: nam eo ipso quod dominus servo peculium constituit. etiam vicario constituisse existimandus est.
} 
Le pécule apparaît initialement pour libérer le maître de la nécessité d'entretenir son esclave et tous le membres de sa famille d'une part, et de l'autre, pour stimuler l'intérêt et l'initiative de l'esclave de mieux administrer le bien qui lui est octroyé, l'aidant à s'entretenir et même à gagner. C'est compréhensible dans la période initiale de l'esclavage classique, lorsque l'esclave n'a aucun statut selon le droit civil, et ne peut, par conséquent, posséder aucun bien propre57. Bien que les Digestes traitent comme personnes sous le pouvoir d'autrui les esclaves, mais aussi les enfants et les petits enfants dépendants de la familia (D. 1.6), cette logique d'instauration d'un bien péculiaire est utilisée à l'égard des deux groupes de personnes indépendamment de leur status libertatis.

La voie de „dématérialisation“ du pécule commence probablement dans une période plus ancienne de la pratique pécuniaire, puisque les Digestes contiennent des textes où le pécule est mentionné comme moyen d'encourager l'épargne et l'initiative des esclaves et des alieni iuris. Ils devraient être examinés dans l'évolution, mais ils caractérisent toute période, toujours dans le contexte de la personne concrète, des intérêts, des dépendances et de l'atmosphère objective. C'est ce qui est souligné dans un texte de Florentius ${ }^{5}$, bien qu'on y tente de généraliser tous les pécules, non seulement ceux à objectifs économiques, mais aussi ceux liés aux revenus

57 IJ.1.16.4 : Servus ... nullum caput habuit. Voir pour la condition et le « statu » des esclaves Buckland. Op.cit. , p.1-72.

$5^{8}$ Pour l'époque où vit Florentinus il y a une discussion - au temps des Antonines (IIe s.) au des Sévères (fin de IIe- début de IIIe s.) Voir l'argumentation chez Petruț-George, B.. Epoca afirmării juristului roman Florentinus. - In : Analele Științifice ale Universității Alexandru Ioan Cuza din Iași. seria Ştiințe Juridice. 2 (2019). p. 171-184. 
dans le cadre d'un service à accomplir, c'est-à-dire le pécule castrense et quasicastrense :

D.15.1.39 (Florentinus libro 11 institutionum) Peculium et ex eo, quod parsimonia sua quis paravit vel officio meruerit a quolibet sibi donari idque velut proprium patrimonium servum suum habere quis voluerit.

C'est pour cette raison que l'instauration de la comptabilité le concernant, respectivement la limitation de la responsabilité jusqu'au montant de sa valeur, sont conçues comme une mesure de protection tant pour son dominus/ pater familias, que pour l'esclave lui-même et pour le fils de famille. Il ne faut pas oublier que dans l'époque reculée de la création de la pratique péculiaire cette règle était en vigueur comme très ancienne, bien que reflétée beaucoup plus tard par Gaius dans son commentaire sur l'édit prétorien, figurant dans les Digestes dans le livre L, titre XVII des Digestes (De diversis regulis iuris antiqui) :

D. 50.17.133 (Gaius libro octavo ad edictum provinciale) Melior condicio nostra per servos fieri potest, deterior fieri non potest.

L'évolution ultérieure du pécule permet d'associer les esclaves et les alieni iuris aux échangescommerciales, de les faire participer aux différents contrats, et ceci non pas sporadiquement par un iussus, mais dans la gestion de toute une activité économique.

Il est difficile de déterminer pourquoi dans certains cas ils sont nommés institores ou exercitores, et dans d'autres on instaurait le pécule. Certains auteurs mettent l'accent sur une plus grande confiance dans les aptitudes des alieni iuris, lorsqu'ils sont chargés de la gestion de toute une entreprise ayant une valeur considérable, la responsabilité étant illimitée et pour cause. Le témoignage d'Ulpianus sur une valeur insignifiante du

\section{4}


pécule:(D.15.1.5.3: ...quasi pusilla pecunia sive patrimonium pusillum“) ne pourrait être considéré comme argument à cet égard et nous l'avons d'ailleurs précisé plus haut. Si à une étape initiale le bien pécuniaire représentait une petite somme, utilisée pour une activité commerciale plus restreinte et le pécule était considéré sous son aspect matériel, dans les Digestes il est déjà représenté comme valeur du bien, incluant des objets de toute sorte, des droits et des obligations, c'est à dire une activité économique déployée à plusieurs niveaux. La détermination des restrictions de la responsabilité lorsqu'on opère avec un bien pécuniaire repose sur la même logique économique et juridique dont on se sert dans les sociétés contemporaines à responsabilité limitée, puisqu'on partage le risque entre les participants au contrat et cette pratique représente une bonne garantie de succès.

\section{Merx peculiaris}

Lorsqu'on détermine le bien pécuniaire les sources juridiques avancent au premier plan le terme de „merx peculiaris“.

La conception de la merx peculiaris est ordinairement liée aux textes qui réglementent l'actio tributoria, qui d'une part, figure dans les Digestes dans le livre XIV où est règlementée la responsabilité illimitée du dominus/ pater familias, de même que dans la reconstruction de Lenel de l'Edictum perpetuum ${ }^{59}$. D'autre part, si on fait la comparaison avec l'actio de peculio

59 Selon LENEL, O. Das Edictum Perpetuum. Ein Versuch zu seiner Wiederherstellung $\left(3^{\mathrm{a}}\right.$ edición, Leipzig, Tauchnitz, 1927; reimp. Aalen, Scientia, 2010, p. 272 ss. Cette action se trouve dans le titre XVIII de l'Edit "Quod cum magistro navis, instituere eove, qui in aliena potestate erit, negotium gestum erit”. Selon la réconstruction cet édit prétorien est triplex pour l'actio exercitoria, actio institoria et actio tributoria et parallèlemant pour la responsabilité limitée il $\mathrm{y}$ a un autre édit triplex: pour actio de peculio, actio de in rem verso et actio quod iussu (D.15.1.1.1). Voir la réconstruvtion de la formule de cette action chez VALIÑO, E. La "actio tributoria". - In : SDHI., 33 (1967), p. 128 ; CHIUSI, T. Contributo allo studio dell'editto "De 
dans le livre XV, là aussi il y a une limitation de la responsabilité, bien que non tellement définitive.

Une grande partie des cas mentionnant la merx peculiaris, ont trait à la réglementation spécifique de la procédure d'insolvabilité du pater familias, lorsque, avant de procéder à la procédure de bonorum venditio ${ }^{60}$, on collecte les créances des débiteurs et on met à part les créances privilégiées des créanciers, le pater familias pouvant être un d'eux. Certains auteurs ${ }^{61}$ notent que c'est bien dans ce dernier cas que l'on trouve l'hypothèse particulière sur l'insolvabilité des biens séparés (appelés dans la doctrine des patrimoines spéciaux ou autonomes pour certaines activités ou ciblés) liés à l'activité commerciale des alieni iuris ${ }^{62}$. Ils sont examinés en détail dans D. 14.4., un titre entier étant consacré à l'actio tributoria, mais dans la plupart des cas on fait la distinction entre leur application et l'application de l' actio de peculio ${ }^{63}$,

tributoria actione”. - In : Atti della Accademia Nazionale dei Lincei : Memorie. Ser. IX, vol. III, fasc. 4 (1993), p. 377 ss.; MICELI, Studi sulla struttura formulare..., cit., p. 355 ss. .

60 Généralement sur le thème, voir PÉREZ ÁLVAREZ, M. del Pilar, La "bonorum venditio". Estudio sobre el concurso de acreedores en Derecho romano clásico.Madrid, 2000, p. 73 ss.; SOZA, Ma DE LOS ÁNGELES, Procedimiento concursal. La posición jurídica del "bonorum emptor" . Madrid, 2008, p. 37 ss.

61 Voir LAZO, P. La "merx peculiaris" como patrimonio especial .. - In : Revista de estudios histórico-jurídicos , 35 (2013), p. 180 ss.; ID. La determinación de la merx peculiaris como etapa previa a su reparto. -In : Revista de Derecho , 1 (2016), p. 95-109. https://revistaderecho.ucn.cl/index.php/revista-derecho/article/view/1798

62 Selon GUARINO, A. Diritto privato romano. Napoli, 2001, p. 411 s. il y a une connexion incontestable entre incompatibilité et tributio. Voir AUSSI PESARESI, Ricerche sul peculium imprenditoriale, cit, p. .

63 Voir MAZZOLENI, A. D.14.4.1.1 e il concetto di merx peculiaris nella disciplina edittale dell'actio tributoria. - In : Forum historiae iuris, https://forhistiur.de/2016-11-mazzoleni/ et spécialement n.1où il y a une bibliogtaphie pour actio tributoria. 
en considérant le privilège du propriétaire du bien péculiaire et en appliquant à son égard le principe „Melior est conditio peculio occupantis“ 64.

Dans les études romanistes on mène une discussion sur l'autonomie de la merx peculiaris - dans quelle mesure elle constitue un bien autonome. La majorité des auteurs sont d'avis qu'il s'agit d'une structure qui s'approche de la conception moderne du capital chez les sociétés commerciales ${ }^{65}$. Une grande partie des arguments sont généralisés dans les ouvrages de SUÁREZ BLÁZQUEZ, qui cite les recherches des autres auteurs de même avis au de contraire ${ }^{66}$. Il admet que la communauté des intérêts est à la base de la

64 Voir SUÁREZ BLÁZQUEZ, G. Ocupación e intervención..., cit. p.313 ss.

65 Voir par ex. la définition dans le manuel de DAZA MARTÍNEZ, J., RODRÍGUEZ ENNES, L. Instituciones de Derecho romano.Valencia, 2009, p. 50: „El peculio es un capital que el esclavo, consintiéndolo el dueño, se forma con las propias fuerzas o donativos. En otras ocasiones es el mismo dueño quien constituye con sus propios bienes un peculium al esclavo, con el fin de que realice una actividad comercial en su favor“.

66 Voir SUAREZ BLÁZQUEZ, G. Dirección y administración de empresas en Roma. Ourense, 2001, p. 47-59, qui admet le pécule comme une structure séparé du patrimoine du pater familias et les esclaves et le fils de famille comme des organes directives de cet entreprise (el ente tiene y desarrolla mediante órganos directivos, como sus hijos y esclavos sujetos a potestad). Ce thèse est développé dans les livres et les articles nombreuses du même auteur : SUÁREZ BLÁZQUEZ, G. Ocupación e intervención judicial de la empresa..., cit.; ID. Naturaleza jurídica de la actio tributoria. - In : RGDR, 20 (2013) ; ID. Intervención judicial de la empresa y de los holding de empresas peculiares comerciales de responsabilidad limitada, en suspensión de pagos, en la Roma clásica. -In : Anuario de la Facultad de Derecho de Ourense, 1(2011), p. 413-430; ID. El Peculio, Ente Jurídico Autónomo Matriz de la Responsabilidad Patrimonial del Empresario : Dueño y los Acreedores sobre la "Merx Perculiaris". - In : Investigación : cultura, ciencia y tecnología, 5 (2011), p.55-58; ID. Graduación de la responsabilidad civil del empresario y de los grupos de empresas -matrices y filiales- de Roma frente a terceros. -In : Revista de derecho : División de Ciencias Jurídicas de la Universidad del Norte, 36 (2011), p. 279-296 ; ID. El peculio como ente jurídico autónomo y matriz de la "merx peculiaris"., cit. p. 119-125 ; ID. Cosideraciones sobre la dirección y dirección de empresas en Roma siglo II a.C. Siglo III d.C. -In : Investigación : cultura, ciencia y tecnología, 2 (2009), p. 64-67 ; ID. Direito de empresas en Roma : projecçáo no direito das empresas actual. -In : Anuario de la Facultad de Derecho de Ourense, 1 (2008), p. 483-663 ; ID. Concurso mercantil de acreedores: "vocatio in tributum". La "acción tributoria" frente al 
structure autonome du pécule, comprenant des droits et des obligations, et non des objets concrets ${ }^{67}$. À partir de la fin de la République, selon lui, le pécule est déjà une structure autonome et affirmée ayant une importance juridique, avec un objectif fondamental - la création d'entreprises commerciales à responsabilité limitée, c'est-à-dire indépendantes du maitre et de ses entreprises, y compris de celles dans lesquelles il participe lui-même. Il est instauré pour que l'on puisse à travers lui participer au commerce. Les alieni iuris deviennent des gérants de l'entreprise commerciale du maître et passent des contrats avec de tiers personnes sans que le maître le sache. La responsabilité limitée apparaît pour protéger ses intérêts, mais aussi ceux des tierces personnes, ayant respectivement une incidence sur la concurrence des créanciers en cas l'actio tributoria si elle est inapplicable. Les préteurs cherchent une protection adéquate des intérêts de tous les participants dans ces relations et le juge assermenté décide lors du procès s'il doit octroyer ou non les privilèges prévus dans l'édit au maitre - propriétaire du bien pécuniaire ${ }^{68}$.

Cette position est soutenue plus au moins catégoriquement et par analogie aux notions et instituts modernes par d'autres auteurs ${ }^{69}$. C'est la définition de Tubero sur le pécule qui sert d'argument principal ( D.15.1.5.4), accepté par Pomponius dans son commentaire sur le droit civil (D. 15.1.4. pr.),

dueño-empresario, y sus directivos-esclavos, -empresarios corruptos. -In : Anuario de la Facultad de Derecho de Ourense, 1(2006), p. 503-524.

67 Вж. SUÁREZ BLÁZQUEZ, G. El peculio como ente jurídico..., cit., p. 122 ss.

68 On cite les opinions et les commentaires de Quintus Mucius, Tubero, Labeo, Celsus, Pedius, Florentinus, Marcianus, Papirius, Pomponius et Ulpianus en D. 15. 1. 4.; D. 15. 1. 5. 1.; D. 15. 1. 6.; D. 15. 1. 7. 5.; D. 15. 1. 32.; D. 15. 1. 39. ; D. 15. 1. 40.; D. 15. 1. 40. 1. Voir SUÁREZ BLÁZQUEZ, G. Ocupación e intervención judicial de la empresa..., cit. p. 674-675.

69 Voir SERRAO. Op. cit., p. 20 ss. .

\section{8}


mais aussi dans certains autres textes ${ }^{70}$. Ils montrent la séparation sur le plan de la comptabilité des deux patrimoines, bien que l'esclave ou le fils dépendant ne puisse, selon le droit civil, être titulaire d'un bien propre. Dans les nombreuses hypothèses examinées par les juristes classiques qui se réfèrent, eux aussi, aux points de vue de leurs prédécesseurs, le pécule constitue un ensemble compliqué de relations issus de toute sorte de contrats avec des biens (ex peculiari re ) ou avec ce qui est inclus dans le pécule ou bien ce qui est acquis avec lui (ex peculio, in peculiari causa, peculii nomine), c'està-dire on opère avec des objets et on crée des obligations, les alieni iuris devenant ainsi des créanciers, de même que des débiteurs en leur nom ou au nom de leur pater familias/dominus, y compris de contrats mortis causachez legatum peculii ${ }^{71}$.

Sur la base de cette revue généralisée des sources Titiana Chiusi conclut que la merx peculiaris ne constitue pas un bien fiduciaire, mais un ensemble d'objets qui a avant tout une importance de procédure et concerne la responsabilité lors de la passation de contrats avec un tel bien ${ }^{72}$.

A cette thèse de Chiusi on oppose d'habitude l'interprétation idéologique des témoignages dans les sources qu'il y a lieu de limiter73. On souligne qu'il existe à Rome dans la période dans laquelle est créé le régime des actiones adjecticiae qualitatis, un libéralisme économique considérable qui ne connait pas de restrictions normatives. Les instructions dans l'édit prétorien ont en fait un caractère de procédure, mais elles cherchent à rétablir l'équilibre entre les intérêts du dominus/ pater familias comme propriétaire

\footnotetext{
70 D. 15.1.5.3; 15.1.5.4; 15.1.7.3; 15.1.7.4; 15.1.7.5.

${ }^{71}$ Voir CERAMI, PETRUCCI. Op. cit., p. 63 s.; BUCKLAND. Op. cit;, p. 198.

72 CHIUSI. Op.cit., p. 333 ss.

73 Вж. LAZO, P. La “merx peculiaris”, cit., p. 183 s.
} 
du bien péculiaire et les créanciers des contrats avec des alieni iuris qui opèrent non seulement avec lui. En plus dans les deux titres des Digestes (D.14.4 et D.15.1) il y a plusieurs cas dans lesquels on avance comme argument la possibilité de stabiliser le bien des débiteurs pour éviter sa distribution entre les créanciers en donnant satisfaction à ces derniers.

Il y a lieu de faire quelques précisions concernant ce qui précède. Dans la plupart des fragments issus des titres correspondants des Digestes on évoque l'instauration de comptes séparés (rationes domini, rationes peculii) du père de famille/ maître et des alieni iuris/esclaves avec leurs pécules. Ils représentent la valorisation des biens en pécules qui souvent sont appelés « patrimoines réels »74. Un témoignage expresse de Florantinus est, elle aussi, en ce sens dans :

D.15.1.39 (Florentinus libro 11 institutionum) Peculium et ex eo consistit, quod parsimonia sua quis paravit vel officio meruerit a quolibet sibi donari idque velut proprium patrimonium servum suum habere quis voluerit.

Le texte est ordinairement examiné en comparant la notion du pécule comme bien autonome, instauré par l'épargne, comme récompense pour un service rendu ou comme don, mais aussi comme un bien octroyé à un esclave qui le possédera comme un bien propre (proprium patrimonium servum suum habere). Il manque ici la définition moins précise de quasi proprium patrimonii servi/ filii, que l'on rencontre dans d'autres textes ${ }^{75}$.

74 Вж. WATSON, A. The Law of Persons in the Later Roman Republic, Oxford, 1967, p. 178;
BUTI, I. Studi sulla capacitá patrimoniale dei “servi”, Napoli, 1976 ; KIRSCHENBAU, A. Sons,
Slaves and Freedmen in Roman Commerce. Jerusalem - Washington, 1987, p. 33 ss.
75 Вж. по- специално D. 15.1.32. pr. (Ulpianus libro secundo disputationum) ... nam qui cum
servo contrahit, universum peculium eius quod ubicumque est veluti patrimonium
intuetur; D. 15.1.47.6 (Paulus libro quartum ad Plautium) Quae diximus in emptore et
venditore, eadem sunt et si alio quovis genere dominium mutatum sit, ut legato, dotis datione, 
Sans doute la détermination du pécule comme bien fait penser à son autonomie, mais certains auteurs soulignent qu'elle est spéciale ${ }^{76}$. Il y a lieu de clarifier pourtant pourquoi deux termes sont introduits - peculium et merx, et dans quelle mesure il y a une différence entre eux, c'est-à-dire ce qui est autonome et dans quelle mesure la merx peculiaris couvre tout le pécule. Voyons l'opinion d'Ulpianus :

D. 14.4.1.2. (Ulpianus libro 29 ad edictum) . Peculiarem autem mercem non sic uti peculium accipimus, quippe peculium deducto quod debetur accipitur, merx peculiaris, etiamsi nihil sit in peculio, dominum tributoria obligat, ita demum si sciente eo negotiabitur.

Il fait une distinction en déterminant comme pécule ce qui reste du bien octroyé, en prélevant les dettes envers le maitre ${ }^{77}$; mais si l'esclave avait négocié en pleine connaissance de son maitre et si dans le pécule il n'y a rien, alors c'est ce dernier qui assume la responsabilité de l'actio tributoria. C'est aussi l'avis de Gaius, à savoir que si le pécule doit être octroyé ou instauré par approbation ultérieure, alors tout dont se sert l'esclave pour négocier en

quia quasi patrimonium liberi hominis peculium servi intellegitur, ubicumque esset". Une définition plus claire on trouve chez Isidore de Séville, mais qui présente l'évolution finale de l'institut à l'époque de Justinien : Isid., Etym., 5.25.5. Peculium proprie minorum est personarum sive servorum. Nam peculium est quod pater vel dominus filium suum vel servum pro suo tractare patitur.

${ }^{76}$ Voir LAZO, P. La “merx peculiaris”..., cit., p. 185 ss. Et les opinions des autres auteurs y cités. 77 Selon ius civile „servus debere non potest“(D.35.1.40.3), mais il existe des textes où il y a des obligations de l'esclave vers son dominus (quod servus domino debetur en D.35.1.40.3) ou réciproquement (quod dominus debet servo en D.15.1.19.2 et D.15.1.17). Voir la généralisation par rapport au pécule en D. 15.1.41 (Ulpianus libro 43 ad Sabinum) Nec servus quicquam debere potest nec servo potest deberi, sed cum eo verbo abutimur, factum magis demonstramus quam ad ius civile referimus obligationem. Itaque quod servo debetur, ab extraneis dominus recte petet, quod servus ipse debet, eo nomine in peculium et si quid inde in rem domini versum est in dominum actio datur. 
pleine connaissance de son maître est considéré comme la merx peculiaris (IGai. 4. 72: in peculiari merce sciente patre dominove negotietur).

En principe la merx peculiaris est le bien soumis à distribution entre les créanciers. Mais elle ne couvre pas entièrement le pécule, représentant la partie déterminée comme objets concrets ou comme valeur et consacrée à l'activité commerciale ${ }^{78}$. Dans ce cas privilegium deductionis du maître est compréhensible et s'applique uniquement si les contrats avec la merx peculiaris ne sont pas passés avec son accord, avec sa connaissance ou son approbation. Le préteur précise que dans tous les autres cas il est un des créanciers ${ }^{79}$.

Si le pécule est octroyé en entier pour le commerce, alors il est considéré comme la merx peculiaris. Selon Gay la merx peculiaris peut représenter la moitié, un tiers ou moins de la composition du pécule ${ }^{80}$. En cas de concurrence des actions la situation des créanciers avec l'actio de peculio, est plus favorable, puisque cette demande concerne tout le bien pécuniaire, tandis que l'actio tributoria est d'un moindre montant ${ }^{81}$. Ainsi en analysant

78 Voir MAZZOLENI, A. Op. cit., p. 1 ss.
79 Voir le même concept mais plus claire en D. 14.4 .1 (Ulpianus libro 29 ad edictum) pr. Huius
quoque edicti non minima utilitas est, ut dominus, qui alioquin in servi contractibus
privilegium habet (quippe cum de peculio dumtaxat teneatur, cuius peculii aestimatio deducto
quod domino debetur fit), tamen, si scierit servum peculiari merce negotiari, velut extraneus
creditor ex hoc edicto in tributum vocatur.
80 Selon SUÁREZ BLÁZQUEZ, G. El peculio como ente jurídico autónomo..., cit., p. 121 on
compare le pécule avec l'uterus maternel qui abrite merx peculiaris, mais une fois née cette
merx, c.à.d. quand pater familias connait, souhaite et participe au commerce avec celle- là,
alors il est à juste titre traité également avec les autres créanciers dans les transactions avec ce
bien.
81 Voir D. 14.4 .11 (Gaius libro nono ad edictum provincial) Aliquando etiam agentibus expedit
potius de peculio agere quam tributoria: nam in hac actione de qua loquimur hoc solum in
divisionem venit, quod in mercibus est quibus negotiatur quodque eo nomine receptum est:
at in actione de peculio totius peculii quantitas spectatur, in quo et merces continentur. Et 
les textes dans les deux titres des Digestes (D.14.4 et D.15.1) on découvre une sorte de traitement doublé - une fois le pécule est séparé dans la comptabilité du patrimonium domini, et une seconde fois on prélève de lui la merx peculiaris, comprenant des marchandises - des objets meubles, avec lesquels l'esclave ou le dépendant opère avec ou sans la connaissance du maitre.

Plus encore: la merx peculiaris est liée aux activités effectuées à travers elle, mais aussi à l'occasion d'elle - dans D. 14.4.5.5 on admet que l'actio tributoria s'applique pour ce qui est reçu de la transformation de la merx peculiaris, c'est-à-dire d'elle et en rapport et/à l'occasion d'elle: ...per hanc actionem tribui iubetur, quod ex ea merce et quod eo nomine receptum est. On trouve dans les sources des exigences pour tenir des comptes séparés si l'esclave ou le dépendant exerce un commerce différent ${ }^{82}$.

Cependant tous les textes ne contiennent pas des informations sur des comptes séparés (ratio) pour cette partie du pécule, lorsqu'on procède au cours de la distribution du bien entre les créanciers à une estimation (aestimatio). Il est fort possible qu'il s'agisse d'une estimation spéciale d'experts au cours du procès sur toutes les prétentions pour une indemnisation en $\operatorname{argent}{ }^{83}$.

fieri potest, ut dimidia forte parte peculii aut tertia vel etiam minore negotietur: fieri praeterea potest, ut patri dominove nihil debeat. Voir l'interpretation de ce texte en Lazo, P. La "merx peculiaris"..., cit ., p. 187 ss.

${ }^{82}$ Un argument tiré de D.14.4.5.15 (Ulpianus libro. 29 ad edictum). Si plures habuit servus creditores, sed quosdam in mercibus certis, an omnes in isdem confundendi erunt et omnes in tributum vocandi...

83 FUENTESECA DEGENEFFE, M. La litis aestimatio desde la perspectiva del procedimiento formulario romano. - In : AFDUDC, 10 (2006), p. 403-412 ; COLACINO, V., Litis aestimatio. In : NNDI, T.IX, Torino, 1963, p.967-968. 
Là Ulpianus met l'accent sur la caractéristique matérielle de la merx peculiaris, en déterminant qu'elle comprend soit les marchandises, soit la valeur reçue en cas de vente :

D.14.4.5.11. (Ulpianus libro 29 ad edictum) Non autem totum peculium venit in tributum, sed id dumtaxat, quod ex ea merce est, sive merces manent sive pretium earum receptum conversumve est in peculium.

Le terme $m e r x^{84}$ fait penser aux composants matériels du patrimoine et non à sa valeur et on souligne que le prix reçu découle de sa transformation en argent. Dans les sources il est employé le plus souvent dans le sens de marchandise, objet d'emptio- venditio, pemutatio et tout autre contrat à effet d'acquisition de la propriété, en soulignant expressément qu'il s'agit de biens meubles- objet du commerce. Le pécule peut également contenir des immobiliers, des esclaves, des créances etc., et alors la responsabilité dans les contrats concèrnants est soumise à un autre régime de procédure ${ }^{85}$. Il faut pourtant avoir en vue que si les marchandises sont destinées à la negotiatio, toutes les negotiationes ne sont pas réalisées au moyen de vente de merces, c'est-à-dire les merces ne sont pas toujours objet de contrat, mais seulement

\footnotetext{
84 La définition du mot «merx » dans les dictionaires est déterminé par sa signification fondamentale d'une marchandise faisant l'objet de transactions avec un transfert de la propriété. Dans les sources juridiques romaines, il y a une signification plus générale de tout ce qui est payé dans le commerce- D. 13, 3. 4.; 1. § 1 D. 14, 4. 1 pr.- 1. ; D. 18, 1.1 pr.; D. 32. 73.4.; D. 46, 1. 42.; D. 50 , 16. 66 u 207; IGai. IV. 72-u 74 etc. Voir BERGER, A. Op. cit., p. 581.

85 Voir D.50.16.66 où Ulpianus précise que „mercis appellatio ad res mobiles tantum pertinet”. En ce sens on voit la définition du commerce (commercium) comme une échange des biens chez Isidore de Séville: Isid.,Orig.,5.25.35 «Commercium dictum a mercibus quo nomine res venales appellamus“. Mais malgré le status des esclaves comme de res le commesrce des esclaves a un régime spéciale- voir Buckland, Op. cit., p. 39-72.
} 
à un type juste des contrats ${ }^{86}$. Ainsi les sources font une distinction entre negotiator qui est un commerçant en sens général, et le mercator qui achète des marchandises dans le but de les revendre, et, par conséquent, l'interprétation de la merx peculiaris doit en tenir compte ${ }^{87}$.

Buckland parle de propriété réelle sur une multitude d'objets, conçus comme universitas et présentés sous le terme de merx peculiaris ${ }^{88}$. Ceci pose la question de la construction juridique qui correspond à ce bien. Dans $D$. 14.4.5.5 on admet que l'actio tributoria s'applique pour ce qui est reçu suite à la transformation de la merx peculiaris, c'est-à-dire d'elle et en rapport et à l'occasion d'elle: ... per hanc actionem tribui iubetur, quod ex ea merce et quod eo nomine receptum est. Ce texte donne raison à l'affirmation sur le caractère autonome de ce bien. ${ }^{89}$. Cependant le traiter comme une universitas ne s'oppose pas de tout à l'idée de son caractère spécial, puisqu'il est traité comme valeur uniquement au cours du procès, sa séparation comptable étant nécessaire alors, surtout s'il ne couvre pas en entier le pécule. De sorte que si nous pouvons en général appliquer les notions contemporaines sur le capital et sur le patrimonium autonome et spécial dans le sens d'un ensemble

86 Вж. CHIUSI, T.Op. cit., p. 317. Parfois on utilise le concept contemporain de droit de commerce aussi pour les pratiques commerciales et sa règlementation en Rome antique. Voir FADDA, C. Istituti commerciali del diritto romano. Introduzione. Napoli, 1987, p. 54 ss.

87 Pour les différences voir MAZZOLENI, A. Op. cit., p. 7-8. Il admet que Ulpianus adhère à l'opinion de Pedius en D.14.4.1.1. que merx peculiaris fait objet des touts les types de contrats sans exception. Mais on suppose une discussion parmi les jurisconsultes romaines et on mention les exeptions pour les pécules de esclaves- fabricants de tissus, tailleurs et tisserands et aussi pour des marchands d'esclaves (servos fullones vel sarcinatores vel textores vel venaliciarios).

88 Вж. BUCKLAND. Op. cit., p. 188, qui la détermine comme « de whole 'property' (de facto) of the slave, and thus has at least in form the character of a universitas » .

${ }^{89}$ Voir l'interprétation de $D$. 14.4.5.11. en ANDRÉS SANTOS, F. Subrogación real y patrimonios especiales en el Derecho romano clásico. Valladolid, 1997, p. 202 et la discussion y citée. 
d'objets, mais aussi de droits et d'obligations, c'est plutôt à l'égard du pécule, que de la merx peculiaris.

S'il faut conclure concernant la merx peculiaris, le maitre/ père de famille :

- a le privilège de séparer ces objets du pécule instauré avant que les autres créanciers ne reçoivent leurs créances des contrats passés avec un bien péculiaire si les alieni iuris avaient opéré avec la merx peculiaris sans sa connaissance (D. 14.4.1: quippe cum de peculio dumtaxat teneatur, cuius peculii aestimatio deducto quod domino debetur)

- participe comme créancier externe lors de la distribution de cette partie du pécule entre les créanciers en cas d'insolvabilité, quand les contrats avaient été passés sans sa connaissance $(D$. 14.4.5.7., IGai. 4.72);

- fait seul la distribution de ce bien entre les créanciers, en collectant les créances et en payant ce bien (D. 14 .4.7.2);

- sa responsabilité réelle est limitée - duntaxat merces peculiares (D. 14.4.5.11.).

Certains auteurs, en défendant l'autonomie du pécule comme capital, se réfèrent à un texte qui compare le pécule à l'homme :

D. 15.1.40 (Marcianus libro quinto regularum) pr. Peculium nascitur crescit decrescit moritur, et ideo eleganter Papirius fronto dicebat peculium simile esse homini.

Extractant du contexte, ce point de vue de Marcianus qui cite Papirius Fronto (juriste de la fin du II s.) ne pourrait être accepté comme indication pour une notion juridique du pécule, c'est-à-dire le concevoir comme une personne morale. Les romains créent un régime pour les différents types de 
personnes morales et non une notion générale et une terminologie spaciale. $\mathrm{Vu}$ le statut des personnes auxquels est octroyé le pécule (esclaves et alieni iuris) il est encore moins possible de penser à une conception pareille, trop modernisée d'ailleurs. Tout ce qui est lié à l'instauration du bien péculiaire doit être interprété dans le contexte de l'utilitas, qui est la force motrice pour la création du droit prétorien, les actiones adiecticiae qualitatis coordonnant l'utilitas publica avec l'utilitas privata 90 .

Il ne faut pas oublier en plus que toute la réglementation sauvegardée dans les Digestes, a trait non pas à la détermination positive du pécule comparé aux autres instituts, classifications et normes matérielles juridiques, mais aux moyens de procédure pour protéger le maitre et les tiers personnes. Vu les particularités de la conception juridique romaine sur les actiones, toute l'information concernant un institut juridique passe toujours par le processus civil, tandis que pour les procès sur la responsabilité dans les contrats commerciaux et dans ceux sur l'insolvabilité des débiteurs, tout bien est traité avec son équivalent en valeur. Il me semble pourtant qu'en dehors de cette logique chercher des parallèles avec les instituts et les conceptions actuels typiques pour les sociétés commerciales, n'est pas fondée. La pratique réelle romaine contient des formes organisationnelles et une réglementation juridique étonnantes pour nous, compte tenu du temps et des conditions de leur création et application, mais elles devraient être interprétées à la lumière de leurs $\mathrm{c}$ onceptions modernes en droit.

${ }^{90}$ D. 1.1.7. 1. (Papinianus libro secundo definitionum) Ius praetorium est, quod praetores introduxerunt adiuvandi vel supplendi vel corrigendi iuris civilis gratia propter utilitatem publicam. Voir aussi D.14.1.1. pr. (Ulpianus libro 28 ad edictum) Utilitatem huius edicti patere nemo est qui ignoret. 


\section{Concessio peculii et les modes de création du pecule.}

L'instauration du bien péculiaire compris comme une valeur ; c. à d. sa valorisation devait précéder l'octroi réel des objets correspondants, les biens ou l'argent, qui sont voir été cédé par le dominus/ pater familias à l'esclave ou au fils de famille. Il y a lieu de préciser dans ce cas que on donne des pécules aux personnes des deux sexes tant pour les esclaves que pour les alieni iuris - membres de la famille, tous désignés comme personae in potestatem, y compris même des esclaves d'autrui donnés par des causes différentes au maître instaurant le pécule (servi fructuarii vel usuarii dans D.15.1.2.) ${ }^{91}$.

L'acte d'octroyer un bien dans un but déterminé comme pécule est ordinairement appelé concessio peculii, bien qu'on emploie dans les sources plutôt le verbe concedere, dont le sens principal est „octroyer, céder“, sans qu'il y ait un transfert de propriété ou instauration de iura in re aliena. Il reflète cependant exactement le sens de céder des objets du patrimonium du maître pour les administrer comme pécule. Ainsi et vu les particularités de la réglementation du pécule dans l'hypothèse d'une actio de peculio il y a lieu d'affirmer qu'en fait le pécule a une caractéristique réelle et son évaluation se fait dans le but d'une éventuelle actio de peculio et l'indemnisation en argent à l'encontre du maître - propriétaire civil du bien péculiaire. Il est difficile d'accepter une position unie des juristes romaines concernante ce qui est appelé « dématérialisation » du pécule et sa détermination comme un capital,

${ }^{91}$ D. 15.1. 1 (Ulpianus libro 29 ad edictum) 3. De eo loquitur. non de ea: sed tamen et ob eam quae est feminini sexus dabitur ex hoc edicto actio. 4. Si cum impubere filio familias vel servo contractum sit. ita dabitur in dominum vel patrem de peculio. si locupletius eorum peculium factum est. 5. Potestatis verbum communiter accipiendum est tam in filio quam in servo 
encore moins comme structure proche du concept actuelle de la personne morale.

Sur la base des déterminations du pécule, citées plus haut, une casuistique est développée sur la volonté expresse du maître par laquelle est instauré le pécule. Dans les comédies de Plautus on parle souvent d'acquisition de biens considérables par des esclaves et des alieni iuris sans la connaissance du maitre/père de famille qu'ils traient comme propres, en volant parfois un bien du pater familias/dominus et en comptant dans les deux cas qu'il va sanctionner ultérieurement cette «auto instauration »du bien volé comme pécule. Probablement pour s'opposer à cette pratique vicieuse on trouve dans les Digestes des exigences de ne pas traiter comme un pécule des biens instaurés réellement sans la connaissance ou sans un acte spécial du maître:

D. 15.1.4 (Pomponius libro septimo ad Sabinum) pr. Peculii est non $i d$, cuius servus seorsum a domino rationem habuerit, sed quod dominus ipse separaverit suam a servi rationem discernens: nam cum servi peculium totum adimere vel augere vel minuere dominus possit, animadvertendum est non quid servus, sed quid dominus constituendi servilis peculii gratia fecerit.

Visiblement dans ce cas on est en présence d'une instauration réelle par des actions concrètes, respectivement avec inscription dans le codex accepti et expensi, probablement avec un autre document contenant un inventaire des objets octroyés comme pécule, et non seulement leur valeur comptable. Cela a dû être nécessaire pour une estimation adéquate du bien pécuniaire au moment de son instauration, respectivement pour la détermination des limites de la responsabilité pour les contrats passés avec lui par les alieni iuris. 
Dans D. 15.1.5.4 Ulpianus citant la définition de Tubero, appuyée par Celsus, offre une autre option- que l'esclave décrive lui-même et instaure le bien comptable, inclus dans le pécule, mais avec la permission du maître (servus domini permissu separatum a rationibus dominicis habet, deducto inde si quid domino debetur). Ceci peut être interprété dans le sens d'une comptabilité à part que l'esclave mène pour des biens qu'il ne soient pas sa propriété, mais avec lequels il opère.

La manifestation active de la volonté expresse du maître ou au moins la connaissance sur l'instauration du bien peculiaire est soulignée par Pomponius, en montrant ainsi la nécessité de l'expression de la volonté du dominus :

D.15.1.4.2. (Pomponius libro septimo ad Sabinum) Ex his apparet non quid servus ignorante domino habuerit peculii esse, sed quid volente: alioquin et quod subripuit servus domino, fiet peculii, quod non est verum.

De toute évidence la pratique est variée dans les conditions d'un grand dynamisme lors de la création et de la mise en œuvre des pécules, et c'est pour cette raison que Pomponius admet une approbation ultérieure d'un bien établi et instauré réellement par l'esclave lui-même, le pécule étant visiblement agréé en ce moment- là :

D.15.1.49 (Pomponius libro quarto ad Quintum Mucium) pr. Non solum id peculium est, quod dominus servo concessit, verum id quoque, quod ignorante quidem eo adquisitum sit, tamen, si rescisset, passurus erat esse in peculio.

Gaius de son côté conclut que même si le pécule est établi réellement sans un acte spécial du maitre, il représente tout ce dont se sert l'esclave pour commercer avec la connaissance de son maitre (IGai. 4,72: in peculiari merce sciente patre dominove negotietur). 
Ulpianus tient compte dans son commentaire relatif à l'édit prétorien, de la réalité, en examinant certaines hypothèses plus spécifiques de concessio peculii. Dans le premier cas il souligne qu'il ne suffit pas que le maître désire certaines actions, il ne devrait pas prohiber ou exclure certaines autres, surtout si la libera administratio peculii est octroyée - probablement par analogie aux prohibitions dans praepositio de l'institor ${ }^{92}$.

La seconde hypothèse est plus intéressante- là le pécule est instauré par le maitre avant qu'il ne devienne fou (furiosus) ou par le pater familias, dont l'héritier est un fils mineur (pupilus). Dans les deux cas il n'est pas possible d'exprimer une volonté juridiquement valable, mais puisque le pécule est déjà établi, donc il existe, à moins qu'il ne soit pas repris, comme précise Marcelus, cité par Ulpianus 93.

Indépendamment des modes d'instauration du pécule la pleine liberté d'opérer avec lui est notée expressément dans „libera administratio peculii“:

D.15.1.7.1. (Ulpianus libro 29 ad edictum) ... Alia causa est peculii liberae administrationis : nam haec specialiter concedenda est.

Par conséquent si le pécule est conçu comme étant un privilège pour le maitre, il doit être indubitablement établi. Pareille exigence est présente pour iussus ${ }^{94}$, mais aussi pour praepositio de l'esclave et du dépendant comme institor ${ }^{95}$. Les sources contiennent différentes hypothèses concernant

92 D.14.4.1.3. (Ulp.1ib.19 ad ed.)...non enim velle debet dominus sed non nolle ...

93 Voir D.15.1.7.1., in fine «...Ego autem puto non esse opus concedi peculium a domino servum habere. sed non adimi. ut habeat ».

94 D.15.4.11 ...iussus autem accipiendum est. sive testato quis sive per epistulam sive verbis aut per nuntium sivve specialiter in uno contracto iusserit sive generaliter.

95 Voir la situation où on confide une taberna (boutique, atelier, ou pub) et on suppose toujours la function d'instrtor sauf le cas où une inscription expresse en latin ou en grec selon le lieu dit que cela est un pécule- alore on donne actio de peculio : D.14.3.11.3 (Ulpianus libro 28 ad edictum) Proscribere palam sic accipimus claris litteris, unde de plano recte legi possit, ante 
cette instauration, ce qui en fin de compte suppose la détermination des limites de la responsabilité du maître: présentation expresse tant d'un inventaire, qu'une connaissance, accord, accès, non opposition, permission, approbation ultérieure des contrats avec de tierces personnes.

L'instauration du pécule met au premier plan la comptabilité correctement menée par le maître et les preuves fournies dans le codex accepti et expensi ${ }^{96}$, certains textes insistant sur la nécessité d'un tel registre de la part non seulement des esclaves et des alieni iuris avec pécule, mais des vicaires aussi.

Ainsi nous pouvons conclure que la création de la responsabilité limitée du maître à l'égard des contrats, ainsi qu'en cas d'insolvabilité liée au pécule, est le résultat d'une longue réforme radicale mise en œuvre au moyen du droit prétorien per utilitatem causa, afin de garantir les échanges commerciaux, conçus dans le sens le plus large à Rome du temps de la République tardive et après. Le pécule s'avère une forme adéquate pour la réalisation de cet objectif, sa forme initiale modeste et sa destination revêtant un nouveau contenu pour se transformer en un moyen adéquat d'entrepreneuriat dans Rome antique.

tabernam scilicet vel ante eum locum in quo negotiatio exercetur, non in loco remoto, sed in evidenti. Litteris utrum Graecis an Latinis ? Puto secundum loci condicionem, ne quis causari possit ignorantiam litterarum. Certe si quis dicat ignorasse se litteras vel non observasse quod propositum erat, cum multi legerent cumque palam esset propositum, non audietur.

96 Pour des contrats littéraux voir ZANDRINO, L. Dalla "pecuna expensa lata" alla "transscriptio" : tracce di una evoluzione del contratto letterale. - In : A Pierluigi Zannini. Scritti di diritto romano e giusantichistici. Milano, 2018, p. 359-379 et la littérature récente ainsi que l'œuvre classique de THILO, R.M. Der Codex accepti et expensi im roemischen Recht, ein Beitrag zur Lehrevon der Literalobligation. Goettingen, 1980 et aussi CREMADES, I. El contrato literal. - In : Derecho romano de obligaciones. Homenaje al profesor Jose Luis Murga Gener. Madrid, 1994, p. 519- 548. 


\section{Conclusion}

La logique de limitation de la responsabilité contractuelle repose, selon les jurisconsultes romains, sur deux circonstances : réalisation d'une activité à travers une autre personne et le caractère de l'activité elle-même, qui admet ou non le contrôle et l'information en temps utile sur les contrats passés. C'est la raison aussi pour faire partager le risque différemment lors de l'instauration du pécule ou lors de présentation d'une préposition, dans l'un des cas on octroie un bien, et dans l'autre, on donne des instructions et on fixe l'étendue des activités, sans tenir compte du chiffre d'affaires avec lequel elles sont réalisées.

Certains auteurs considèrent qu'il est question d'une plus grande confiance et d'une plus grande liberté lorsqu'on nomme un esclave ou un dépendant pour exercitor ou institor, bien que les textes dans lesquels on parle de relations péculiaires ne supposent pas de tout une autre limitation de l'activité des esclaves et des alieni iuris que celle d'opérer avec le bien instauré.

En droit contemporain, de même qu'en droit romain ordinairement le rapport du risque, respectivement des prétentions des entrepreneurs et des créanciers, est réglementé à un niveau normatif. L'édit prétorien, commenté par les juristes, représente la base normative pour adopter des différentes solutions casuistiques en Digestes. Le préteur répond aux nécessités sociales et économiques liées au déploiement d'une activité commerciale et économique la plus large à Rome dans la période de la République tardive et ceci sur un territoire augmentant sans cesse, par le choix de diverses formes juridiques, d'organisation y compris adaptées à la pratique commerciale internationale. Tant pour le pécule que pour la praepositio il est question d'équilibrer les intérêts, de garantir des créances et de donner une 
information claire sur ce qui est permis et limité, qui de son côté conditionne le genre et l'étendue de la responsabilité sur les contrats juridiques passés par les esclaves et les alieni iuris.

La jurisprudence romaine est loin du concept et des notions relatives à l'entreprise commerciale, au capital, à la personne morale etc. du droit moderne, bien qu'il existe des formes d'organisation analogiques dans le commerce romain, en particulier après l'établissement d'une domination politique et économique en Méditerranée dans le cadre de l'Empire romain.

On ne pourrait donc pas accepter inconditionnellement un tel niveau d'autonomie du pécule ou de la merx peculiaris, qui seraient identiques à la notion moderne de capital ou d'une structure, proche à la personne morale par analogie avec hereditas iucens. Cependant le fait d'instaurer au sein du patrimoin du maître/père de famille tant un pécule qu'une merx peculiaris et la réalisation d'une responsabilité limitée jusqu'au niveau de leur équivalent en valeur, constitue une partie du mécanisme, mis en place par les juristes romains de contrôle des risques des entrepreneurs et des créanciers. En fin du compte, basée sur la combinaison entre l'utilitas publica ct l'utilitas privata la pratique prétorienne et les opinions des juristes classiques inclus dans les Digestes, sont orientées vers la synchronisation des intérêts pour arriver à la stabilisation des patrimoines avec lequels on prend part aux opérations commerciales, et pour minimiser les pertes en cas d'éventuelle insolvabilité. 